\title{
Genome-directed Discovery of
}

\section{Tetrahydroisoquinolines from Deep-sea Derived}

\section{Streptomyces niveus SCSIO 3406}

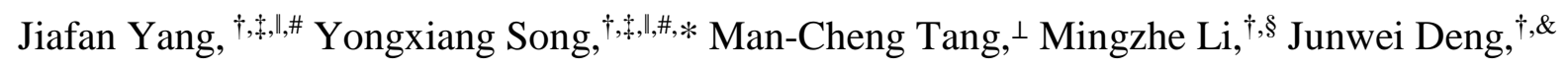
Nai-Kei Wong, ${ }^{\Delta}$ and Jianhua Ju ${ }^{\dagger,+, \|, *}$

${ }^{\dagger}$ CAS Key Laboratory of Tropical Marine Bio-resources and Ecology, Guangdong Key Laboratory of Marine Materia Medica, RNAM Center for Marine Microbiology, South China Sea Institute of Oceanology, Chinese Academy of Sciences, 164 West Xingang Road, Guangzhou 510301, China;

$\$$ University of Chinese Academy of Sciences, 19 Yuquan Road, Beijing 100049, China;

'Southern Marine Science and Engineering Guangdong Laboratory (Guangzhou), No.1119, Haibin Rd., Nansha District, Guangzhou 511458, China

${ }^{\perp}$ State Key Laboratory of Microbial Metabolism, School of Life Sciences \& Biotechnology, Shanghai Jiao Tong University, Shanghai, 200240, China.

${ }^{\S}$ Department of Applied Chemistry, College of Materials and Energy, South China Agricultural University, Guangzhou 510642, China.

${ }^{\&}$ School of Life Sciences, Sun Yat-sen University, Guangzhou 510275, China.

${ }^{\Delta}$ Department of Pharmacology, Shantou University Medical College, Shantou 515041, China

*Corresponding Authors, songx@scsio.ac.cn or jju@sssio.ac.cn

\#These authors J. Yang and Y. Song contributed equally. 


\begin{tabular}{ll}
\hline Table of contents & Page
\end{tabular}

Table S1. The recipes for screening Streptomyces niveus SCSIO $3406 \quad$ S3

Table S2. Comparing the ${ }^{1} \mathrm{H}(700 \mathrm{MHz})$ and ${ }^{13} \mathrm{C}$ NMR (175 HMz) Spectroscopic Data S4-5 of Compound $\mathbf{1}$ and $\mathbf{2}$ with the literature

Table S3. The crystal parameters of compounds 1 and $2 \quad$ S6

$\begin{array}{ll}\text { Figure S1. Three reported types of naturally occurring THIQs } & \text { S7 }\end{array}$

Figure S2. The key COSY, HMBC and NOESY correlations of compound $2 \quad$ S7

Figure S3. The structure of aclidinomycin A (1) showing the atom labeling and 30\% S8 robability displacement ellipsoids.

Figure S4. The structure of aclidinomycin B (2) showing the atom labeling and 30\% S9 probability displacement ellipsoids.

Figures S5-S10. 1D and 2D NMR spectra of $\mathbf{3}$ in $\mathrm{CD}_{3} \mathrm{OD}$ S10-S15

Figures S11-S16. 1D and 2D NMR spectra of 4 in $\mathrm{CD}_{3} \mathrm{OD}$ S16-S21

$\begin{array}{ll}\text { Figures S17-S22. 1D and 2D NMR spectra of } 5 \text { in } \mathrm{CD}_{3} \mathrm{OD} & \text { S22-S27 }\end{array}$

Figures S23-S28. 1D and 2D NMR spectra of 6 in $\mathrm{CD}_{3} \mathrm{OD}$ S28-S33

Figures S29-S34. 1D and 2D NMR spectra of 7 in $\mathrm{CD}_{3} \mathrm{OD}$ S34-S39

Figures S35-S40. 1D and 2D NMR spectra of 8 in $\mathrm{CD}_{3} \mathrm{OD}$ S40-S45

Figures S41-S46. 1D and 2D NMR spectra of 9 in $\mathrm{CD}_{3} \mathrm{OD}$ S46-S51

Figures S47-S52. 1D and 2D NMR spectra of 10 in $\mathrm{CD}_{3} \mathrm{OD}$ S52-S57

Figures S53-S58. 1D and 2D NMR spectra of $\mathbf{1 1}$ in $\mathrm{CD}_{3} \mathrm{OD}$ S58-S63 
Table S1 The recipes for screening Streptomyces niveus SCSIO 3406

\begin{tabular}{|c|c|c|c|c|c|c|c|c|}
\hline & 1 & 2 & 3 & 4 & 5 & 6 & 7 & 8 \\
\hline Recipes (g/L) & Am6-1 & ISP3 & A1 & Hmt & YMS & $\mathrm{N} 4$ & MAM2ab & RA \\
\hline starch & 20 & & 10 & & 4 & 15 & 5 & 20 \\
\hline glycerol & 10 & & & 20 & & 8 & & \\
\hline glucose & & & & & & & 20 & 10 \\
\hline maltose & & & & & & & & 10 \\
\hline Corn powder & & & & & & & & 5 \\
\hline Oat powder & & 20 & & & 5 & & & \\
\hline Soybean powder & & & & & & & 5 & \\
\hline Yeast extract & 5 & & 4 & 5 & 4 & & 2 & \\
\hline peptone & & & 2 & & & 5 & 2 & \\
\hline Malt extract & & & & & 10 & & & 10 \\
\hline Fish protein & & & 10 & 10 & & & & \\
\hline Fish peptone & & & & & & 8 & & \\
\hline $\mathrm{KH}_{2} \mathrm{PO}_{4}$ & & & & & & & 0.5 & \\
\hline $\mathrm{MgSO}_{4} \cdot 7 \mathrm{H}_{2} \mathrm{O}$ & & & & & & & 0.5 & \\
\hline $\mathrm{NaCl}$ & & & & & & & 4 & \\
\hline $\mathrm{KBr}$ & & & & & & 0.2 & & \\
\hline $\mathrm{FeSO}_{4} \cdot \mathrm{H}_{2} \mathrm{O}$ & & 0.1 & & & & & & \\
\hline $\mathrm{MnCL}_{2} \cdot 4 \mathrm{H}_{2} \mathrm{O}$ & & 0.1 & & & & & & \\
\hline $\mathrm{ZnSO}_{4} \cdot 7 \mathrm{H}_{2} \mathrm{O}$ & & 0.1 & & & & & & \\
\hline Element trace & & $1 \mathrm{~mL}$ & & & & & & $100 \mathrm{vL}$ \\
\hline Sea salt & 30 & 30 & 30 & 30 & 30 & 30 & 30 & 30 \\
\hline $\mathrm{pH}$ & $7.2-7.4$ & $7.2-7.4$ & $7.2-7.4$ & $7.2-7.4$ & $7.2-7.4$ & $7.2-7.4$ & $7.2-7.4$ & $7.2-7.4$ \\
\hline $\mathrm{CaCO}_{3}$ & 5 & & & 5 & & 2 & 2 & 2 \\
\hline
\end{tabular}


Table S2. Comparing the ${ }^{1} \mathrm{H}(700 \mathrm{MHz})$ and ${ }^{13} \mathrm{C}\left\{{ }^{1} \mathrm{H}\right\}$ NMR $(175 \mathrm{HMz})$ Spectroscopic Data of Compound 1 and 2 with the literature

\begin{tabular}{|c|c|c|c|c|c|c|c|c|c|}
\hline \multirow[b]{2}{*}{$\begin{array}{l}\text { Po } \\
\text { s. }\end{array}$} & & \multicolumn{2}{|r|}{1 (in $\mathrm{CDCl}_{3}$ ) } & \multicolumn{2}{|r|}{ aclidinomycin $\mathrm{A}^{1}$} & \multicolumn{2}{|r|}{2 (in $\left.\mathrm{CDCl}_{3}\right)$} & \multicolumn{2}{|r|}{ aclidinomycin $\mathbf{B}^{1}$} \\
\hline & & $\delta_{\mathrm{C}}$ & $\delta_{\mathrm{H},}$ mult. $\quad(J$ in $\mathrm{Hz})$ & $\delta_{\mathrm{C}}$ & $\delta_{\mathrm{H},}$ mult. $\quad(J$ in $\mathrm{Hz})$ & $\delta_{\mathrm{C}}$ & $\delta_{\mathrm{H},}$ mult. $\quad(J$ in $\mathrm{Hz})$ & $\delta_{\mathrm{C}}$ & $\delta_{\mathrm{H}, \text { mult. }} \quad(J$ in $\mathrm{Hz})$ \\
\hline 1 & $\begin{array}{l}\mathrm{CH} \\
2\end{array}$ & 48.6 & $\begin{array}{l}2.53(\mathrm{ddd}, J=8.4,7.2,1.8 \\
\mathrm{Hz}) \\
2.24(\mathrm{td}, J=8.4,7.2 \mathrm{~Hz})\end{array}$ & 48.5 & $\begin{array}{l}2.24(\mathrm{dt}, J=9.2,8.6 \mathrm{~Hz}) \\
2.53(\mathrm{ddd}, J=8.3,7.3,1.8 \\
\mathrm{Hz})\end{array}$ & 48.4 & $\begin{array}{l}2.53(*) \\
2.24(\mathrm{q}, J=8.8 \mathrm{~Hz})\end{array}$ & 48.5 & $\begin{array}{l}2.48(\mathrm{~m}) \\
2.24(\mathrm{dt}, J=9.2,8.3 \mathrm{~Hz})\end{array}$ \\
\hline 2 & $\begin{array}{l}\mathrm{CH} \\
2\end{array}$ & 66.0 & $\begin{array}{l}3.78(\mathrm{ddd}, \mathrm{J}=8.3,7.2,1.8 \\
\mathrm{Hz}) \\
3.70(\mathrm{dt}, J=9.5,7.2 \mathrm{~Hz})\end{array}$ & 65.9 & $\begin{array}{l}3.79(\mathrm{ddd}, J=8.4,7.6,1.8 \\
\mathrm{Hz}) \\
3.71(\mathrm{ddd}, J=9.5,7.2 \mathrm{~Hz})\end{array}$ & 66.0 & $\begin{array}{l}3.76(\mathrm{~m}) \\
3.69(\mathrm{t}, J=8.4 \mathrm{~Hz})\end{array}$ & 65.9 & $\begin{array}{l}3.77(\mathrm{ddd}, J=8.3,7.3,1.8 \\
\mathrm{Hz}) \\
3.68(\mathrm{dt}, J=9.2,7.3 \mathrm{~Hz})\end{array}$ \\
\hline $3 a$ & $\mathrm{CH}$ & 91.5 & $3.93(\mathrm{~d}, J=3.7 \mathrm{~Hz})$ & 91.3 & $3.94(\mathrm{~d}, J=3.7 \mathrm{~Hz})$ & 91.3 & $3.94(\mathrm{~d}, J=3.5 \mathrm{~Hz})$ & 91.1 & $3.92(\mathrm{~d}, J=3.8 \mathrm{~Hz})$ \\
\hline 4 & $\mathrm{CH}$ & 37.8 & $2.87(\mathrm{~m})$ & 37.6 & $2.86 \quad(\mathrm{~m})$ & 37.8 & $2.85(\mathrm{~m})$ & 37.6 & $2.85(\mathrm{~m})$ \\
\hline $4^{\prime}$ & $\begin{array}{l}\mathrm{CH} \\
2\end{array}$ & 19.4 & $\begin{array}{l}1.79(\mathrm{ddd}, J=14.1,10.9 \\
7.7 \mathrm{~Hz}) \\
1.68(\mathrm{ddd}, J=14.1,7.7 \\
1.7 \mathrm{~Hz})\end{array}$ & 19.2 & $\begin{array}{l}1.76(\mathrm{ddd}, J=13.8,11.0, \\
7.3 \mathrm{~Hz}) \\
1.69(\mathrm{ddd}, J=13.8,7.3,1.8 \\
\mathrm{Hz})\end{array}$ & 19.3 & $\begin{array}{l}1.79(\mathrm{~m}) \\
1.56(\mathrm{dd}, J=14.4, \\
7.9 \mathrm{~Hz})\end{array}$ & 19.1 & $\begin{array}{l}1.77(\mathrm{ddd}, J=14.0 \\
11.0,7.6 \mathrm{~Hz}) \\
1.56(\mathrm{ddd}, J=14.0,8.0 \\
1.5 \mathrm{~Hz})\end{array}$ \\
\hline $4 a$ & $\mathrm{CH}$ & 62.5 & $3.22(\mathrm{t}, J=5.1 \mathrm{~Hz})$ & & & 62.2 & $3.26(\mathrm{t}, J=5.4 \mathrm{~Hz})$ & & \\
\hline $5^{\prime}$ & $\begin{array}{l}\mathrm{CH} \\
3\end{array}$ & 41.3 & $2.51(\mathrm{~s})$ & 41.1 & $2.52(\mathrm{~s})$ & 41.1 & $2.53(*)$ & 40.9 & $2.51(\mathrm{~s})$ \\
\hline $\begin{array}{l}6 \\
7\end{array}$ & $\begin{array}{l}\mathrm{CH} \\
\mathrm{CH}\end{array}$ & $\begin{array}{l}63.6 \\
91.8\end{array}$ & $\begin{array}{l}3.31(\mathrm{dt}, J=7.0,2.3 \mathrm{~Hz}) \\
5.32(\mathrm{br} \mathrm{q}, J=2.6 \mathrm{~Hz})\end{array}$ & $\begin{array}{l}63.4 \\
91.6\end{array}$ & $\begin{array}{l}3.31(\text { br d, } J=7.3) \\
5.53(\text { br q, } J=2.8 \mathrm{~Hz})\end{array}$ & $\begin{array}{l}63.1 \\
90.0\end{array}$ & $\begin{array}{l}3.37(\mathrm{~d}, J=7.2 \mathrm{~Hz}) \\
5.63(\text { br s })\end{array}$ & $\begin{array}{l}62.9 \\
90.7\end{array}$ & $\begin{array}{l}3.36(\text { br d, } J=7.6 \mathrm{~Hz}) \\
5.59(\text { br s })\end{array}$ \\
\hline 9 & $\mathrm{C}$ & 159.1 & & 159.0 & & 156.5 & & 157.4 & \\
\hline $9^{\prime}$ & & $\begin{array}{l}72.7 \\
\mathrm{CH}_{2}\end{array}$ & $\begin{array}{l}5.41(\mathrm{dd}, J=15.6,2.1 \mathrm{~Hz}) \\
4.97(\mathrm{ddd}, J=15.6,2.7 \\
1.2 \mathrm{~Hz})\end{array}$ & 72.6 & $\begin{array}{l}5.42(\mathrm{dd}, J=15.6,2,8 \mathrm{~Hz}) \\
4.98(\mathrm{ddd}, J=15.6,2.6,1.2 \\
\mathrm{Hz})\end{array}$ & $98.1, \mathrm{CH}$ & $6.46(\mathrm{~d}, J=2.3 \mathrm{~Hz})$ & 97.7 & $6.49(\mathrm{~d}, J=1.5 \mathrm{~Hz})$ \\
\hline $9 \mathrm{a}$ & $\mathrm{C}$ & 96.2 & & 96.0 & & 96.9 & & 96.8 & \\
\hline 10 & $\mathrm{C}$ & 175.6 & & 175.4 & & 176.4 & & 175.8 & \\
\hline 11 & $\mathrm{C}$ & 162.9 & & 162.8 & & 162.6 & & 162.4 & \\
\hline $11^{\prime}$ & $\mathrm{CH}$ & 61.4 & $4.18(\mathrm{~s})$ & 61.2 & $4.19(\mathrm{~s})$ & 61.5 & $4.16(\mathrm{~s})$ & 61.3 & $4.12(\mathrm{~s})$ \\
\hline 12 & $\begin{array}{l}3 \\
\mathrm{C}\end{array}$ & 125.1 & & 124.9 & & 126.1 & & 126.5 & \\
\hline $12^{\prime}$ & $\mathrm{CH}$ & 9.4 & $1.91(\mathrm{~s})$ & 9.2 & $1.92(\mathrm{~s})$ & 9.6 & $1.94(\mathrm{~s})$ & 9.5 & $1.94(\mathrm{~s})$ \\
\hline
\end{tabular}




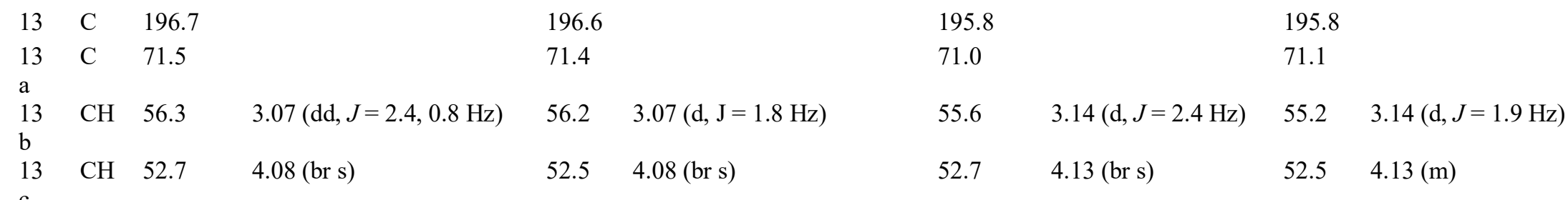

*Responses overlapped.

${ }^{1}$ Cang, S.; Ohta, S.; Chiba, H.; Johdo, O.; Nomura, H.; Nagamatsu, Y.; Yoshimoto, A. J Antibiot (Tokyo) $2001,54,304$. 
Table S3. The crystal parameters of compounds $\mathbf{1}$ and $\mathbf{2}$

\begin{tabular}{|c|c|c|}
\hline & compound $\mathbf{1}$ & compound $\mathbf{2}$ \\
\hline Molecular formula & $\mathrm{C}_{21} \mathrm{H}_{26} \mathrm{~N}_{3} \mathrm{O}_{6.5}$ & $\mathrm{C}_{22} \mathrm{H}_{31} \mathrm{~N}_{3} \mathrm{O}_{9}$ \\
\hline Formula weight & 424.45 & 481.50 \\
\hline Temperature/K & $293(2)$ & $99.9(8)$ \\
\hline Crystal system & orthorhombic & orthorhombic \\
\hline Space group & $P 2_{1} 2_{1} 2_{1}$ & $P 2_{1} 2_{1} 2_{1}$ \\
\hline $\mathrm{a} / \AA$ & $7.74010(10)$ & $7.72610(10)$ \\
\hline $\mathrm{b} / \AA$ & $9.43780(10)$ & $8.79210(10)$ \\
\hline $\mathrm{c} / \AA \AA$ & $26.3562(3)$ & $30.7362(3)$ \\
\hline$\alpha /{ }^{\circ}$ & 90 & 90 \\
\hline$\beta /{ }^{\circ}$ & 90 & 90 \\
\hline$\gamma / 0$ & 90 & 90 \\
\hline Volume $/ \AA^{3}$ & $1925.31(4)$ & $2087.87(4)$ \\
\hline Z & 4 & 4 \\
\hline$\rho_{\text {calc }} \mathrm{g} / \mathrm{cm}^{3}$ & 1.464 & 1.532 \\
\hline$\mu / \mathrm{mm}^{-1}$ & 0.914 & 1.006 \\
\hline $\mathrm{F}(000)$ & 900.0 & 1024.0 \\
\hline Crystal size $/ \mathrm{mm}^{3}$ & $0.300 \times 0.260 \times 0.230$ & $0.27 \times 0.25 \times 0.23$ \\
\hline Radiation & $\mathrm{CuK} \alpha(\lambda=1.54184)$ & $\mathrm{CuK} \alpha(\lambda=1.54184)$ \\
\hline $2 \Theta$ range for data collection $/^{\circ}$ & 9.954 to 148.308 & 10.466 to 148.226 \\
\hline Index ranges & $-9 \leq \mathrm{h} \leq 8,-11 \leq \mathrm{k} \leq 8,-30 \leq 1 \leq 32$ & $-8 \leq \mathrm{h} \leq 9,-10 \leq \mathrm{k} \leq 10,-37 \leq 1 \leq 32$ \\
\hline Reflections collected & 9795 & 9521 \\
\hline Independent reflections & $3792\left[\mathrm{R}_{\text {int }}=0.0311, \mathrm{R}_{\text {sigma }}=0.0327\right]$ & $4088\left[R_{\text {int }}=0.0289, R_{\text {sigma }}=0.0280\right]$ \\
\hline Data/restraints/parameters & $3792 / 0 / 278$ & $4088 / 0 / 317$ \\
\hline Goodness-of-fit on $\mathrm{F}^{2}$ & 1.048 & 1.034 \\
\hline Final $R$ indexes $[\mathrm{I}>=2 \sigma(\mathrm{I})]$ & $\mathrm{R}_{1}=0.0421, \mathrm{wR}_{2}=0.1089$ & $\mathrm{R}_{1}=0.0362, \mathrm{wR}_{2}=0.0969$ \\
\hline Final $\mathrm{R}$ indexes [all data] & $\mathrm{R}_{1}=0.0430, \mathrm{wR}_{2}=0.1096$ & $\mathrm{R}_{1}=0.0371, \mathrm{wR}_{2}=0.0974$ \\
\hline Largest diff. peak/hole / e $\AA^{-3}$ & $0.81 /-1.01$ & $0.33 /-0.31$ \\
\hline Flack parameter & $0.03(8)$ & $0.04(7)$ \\
\hline
\end{tabular}




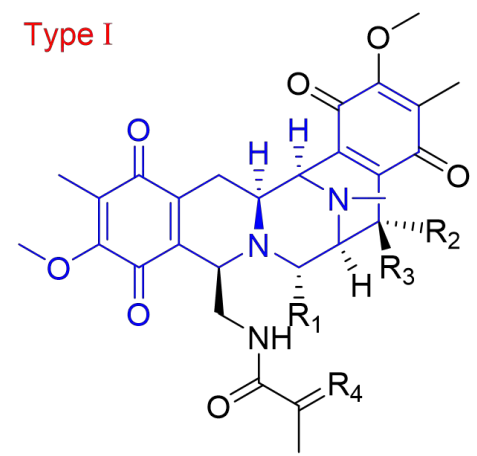

Saframycins

Type II

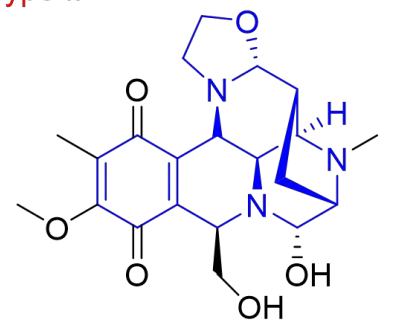

Naphthyridinomycin

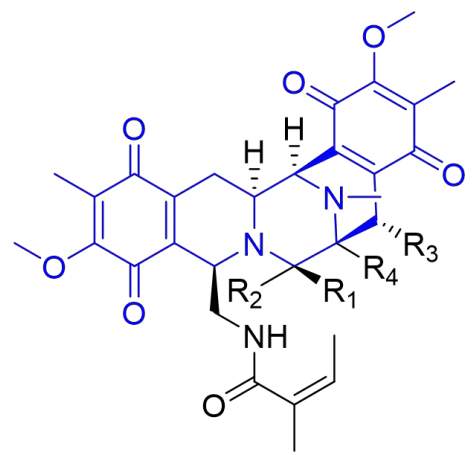

Renieramycins

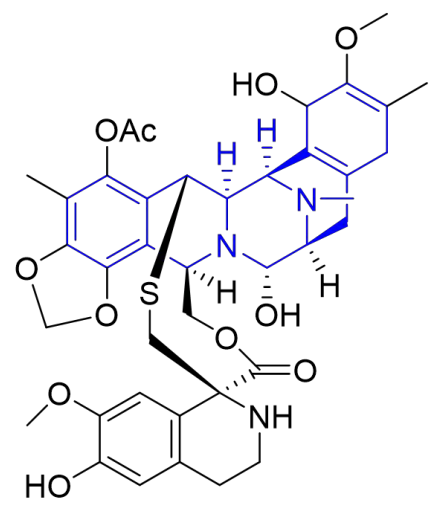

Ecteinascidin 743

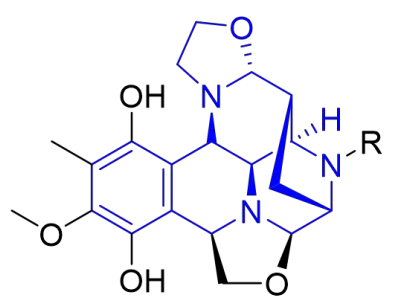

Bioxalomycin $\alpha_{1} \mathrm{R}=\mathrm{H}$ Bioxalomycin $\alpha_{2} R=M e$

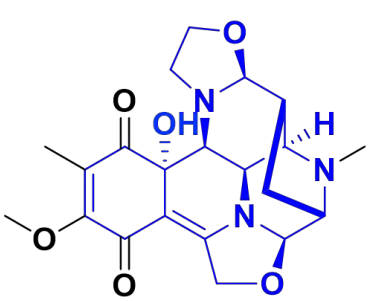

Aclidinomycin A

Type III

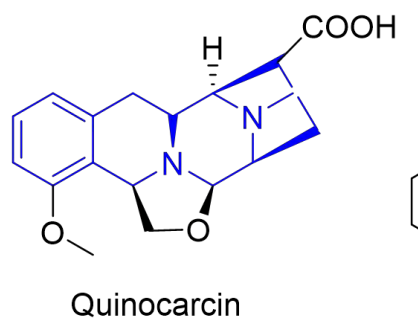<smiles>COc1c(NC(=O)[C@@H]2NCCC[C@H]2O)ccc2c1C1COC3C[C@H]1N1C3CC(CO)[C@H]1C2</smiles>

Tetrazomine

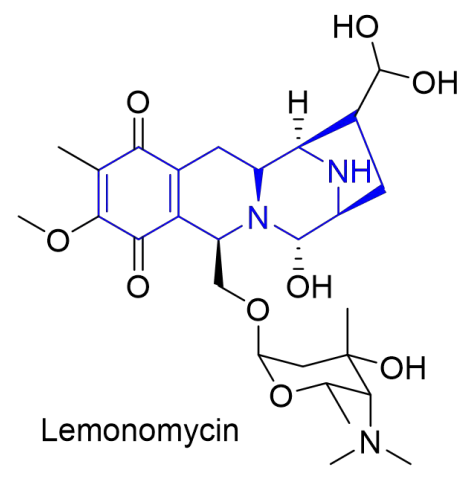

Figure S1. Three reported types of naturally occurring THIQs.

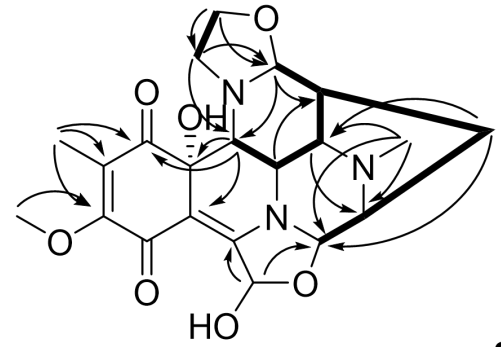

- $\cos \rightleftharpoons \mathrm{HMBC}$

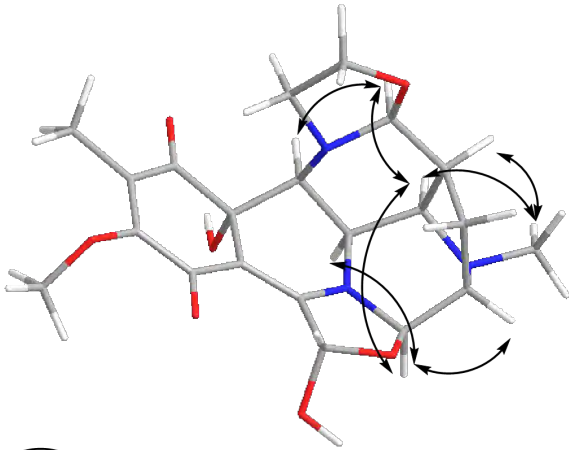

$\frown$ NOESY

Figure S2. The key COSY, HMBC and NOESY correlations of compound 2 


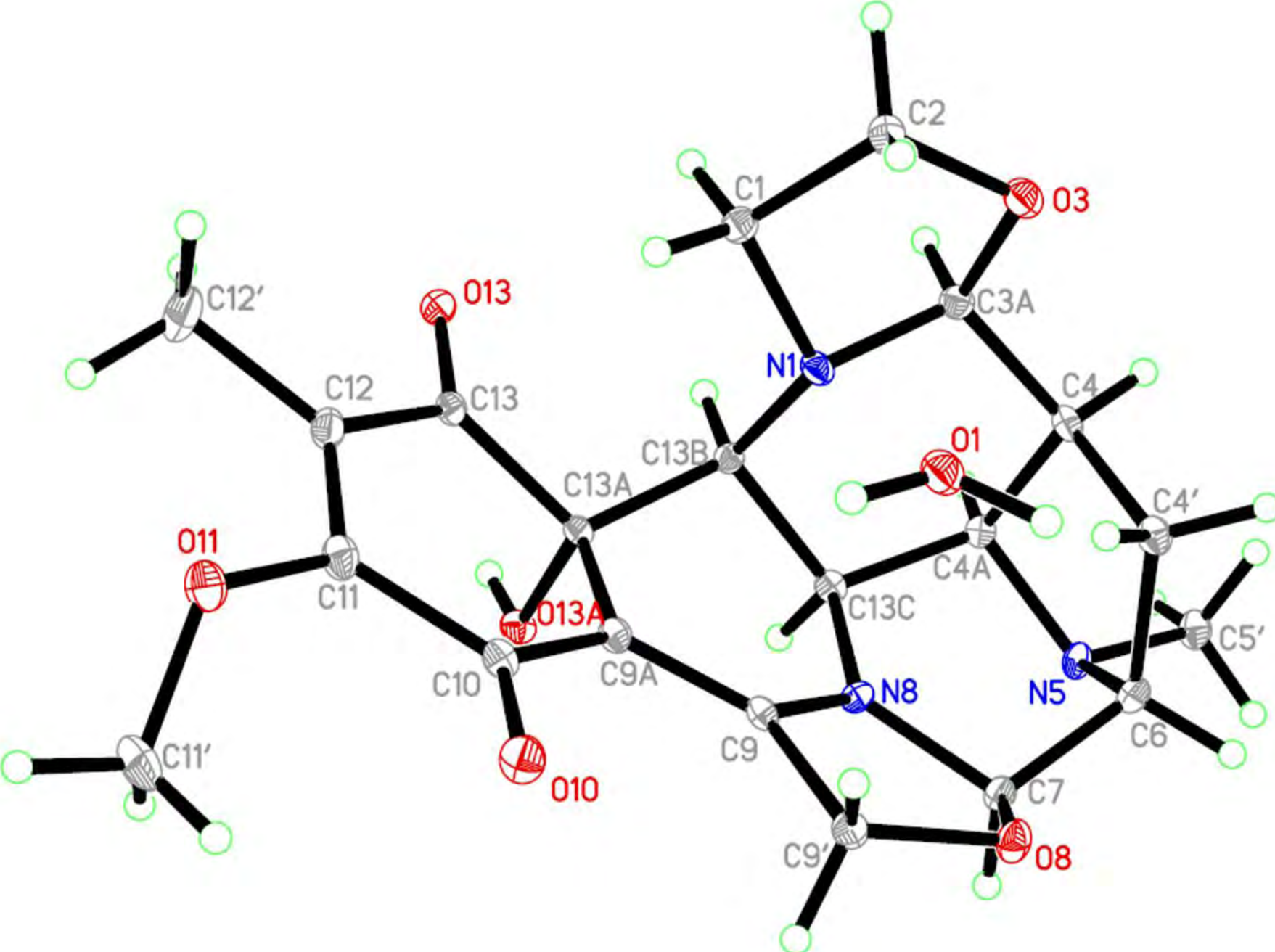

Figure S3. The structure of aclidinomycin A (1) showing the atom labeling and 30\% probability displacement ellipsoids. 


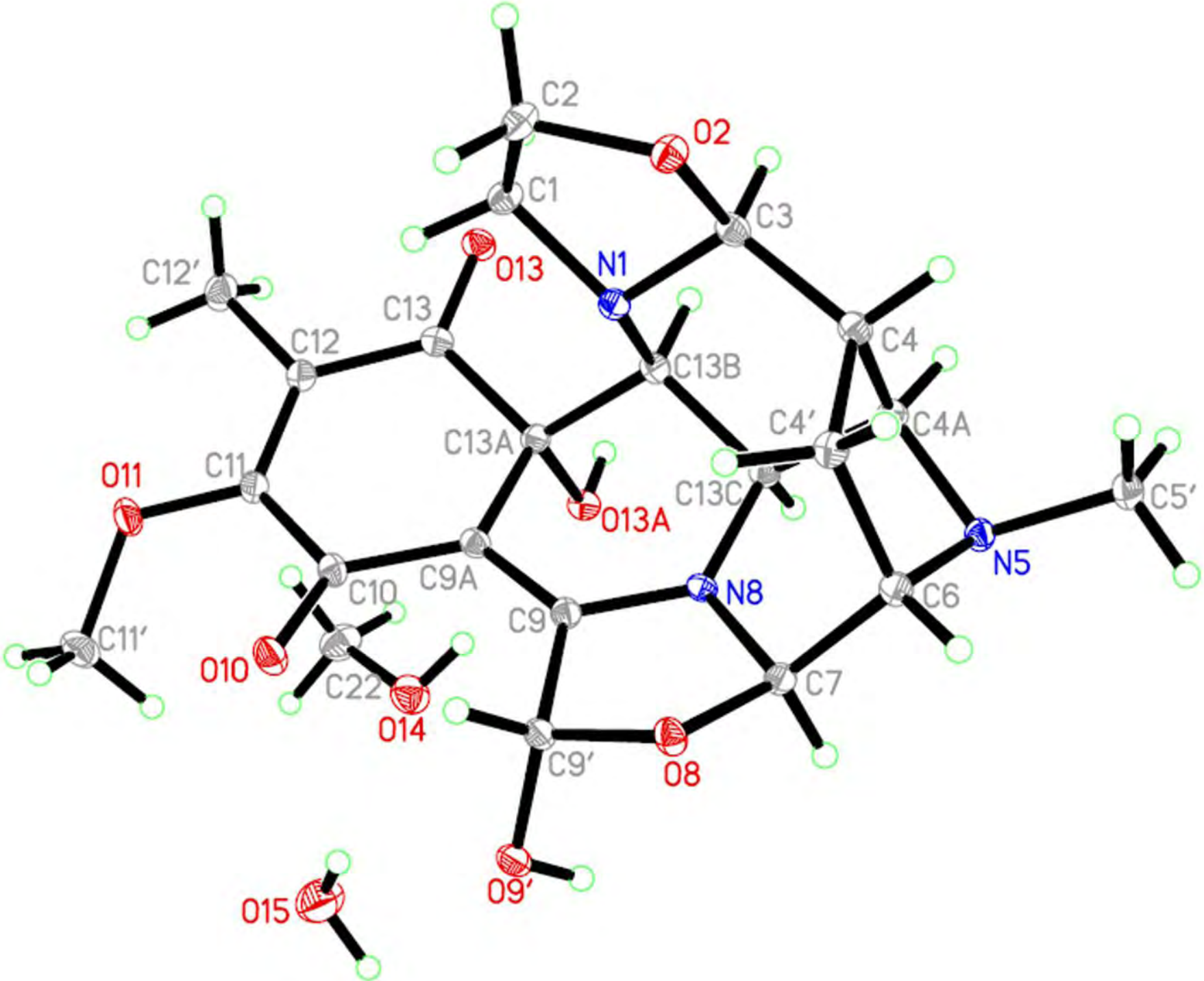

Figure S4. The structure of aclidinomycin B (2) showing the atom labeling and 30\% probability displacement ellipsoids. 
Figure S5. ${ }^{1} \mathrm{H}$ NMR $(700 \mathrm{MHz})$ spectrum of 3 in $\mathrm{CD}_{3} \mathrm{OD}$

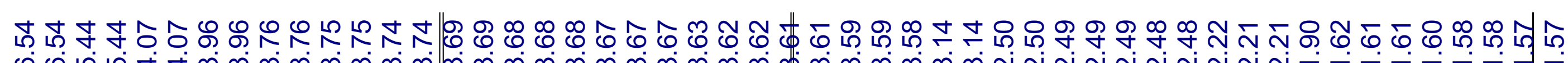

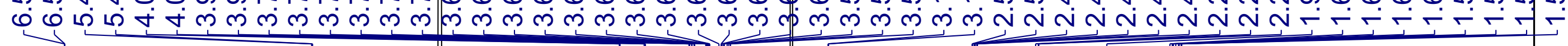
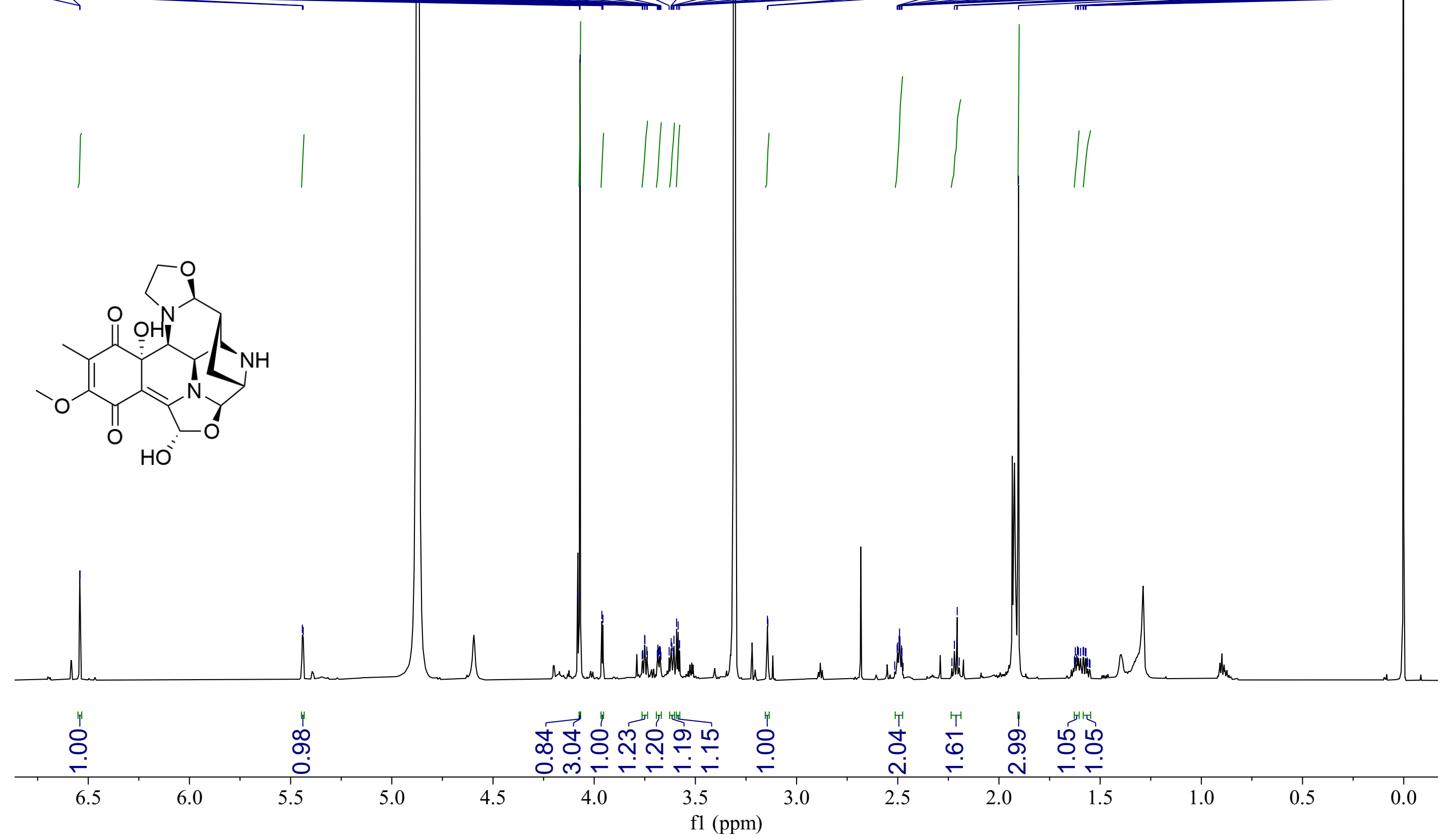
Figure S6. ${ }^{13} \mathrm{C}\left\{{ }^{1} \mathrm{H}\right\} \mathrm{NMR}(175 \mathrm{MHz})$ spectrum of 3 in $\mathrm{CD}_{3} \mathrm{OD}$

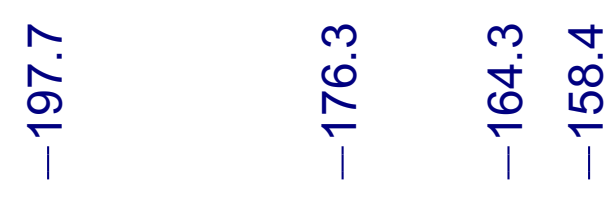

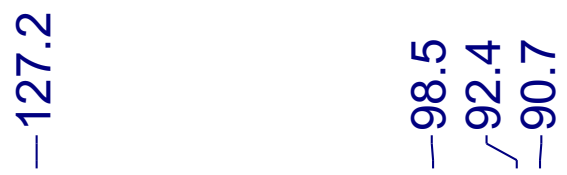

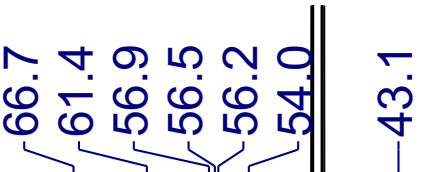

$\stackrel{0}{i} \quad \stackrel{+}{i}$

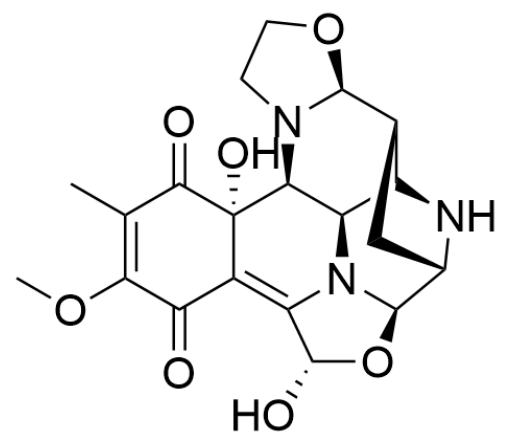

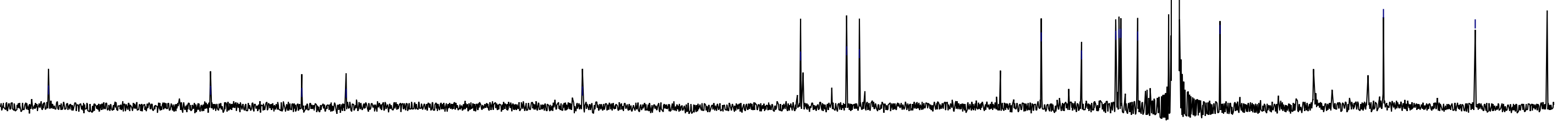

\begin{tabular}{|c|c|c|c|c|c|c|c|c|c|c|c|c|c|c|c|c|c|c|c|}
\hline 200 & 190 & 180 & 170 & 160 & 150 & 140 & 130 & 120 & \multicolumn{2}{|c|}{$110 \quad 100$} & 90 & 80 & 70 & 60 & 50 & 40 & 30 & 20 & 10 \\
\hline
\end{tabular}


Figure S7. $\mathrm{HMQC}$ spectrum of 3 in $\mathrm{CD}_{3} \mathrm{OD}$

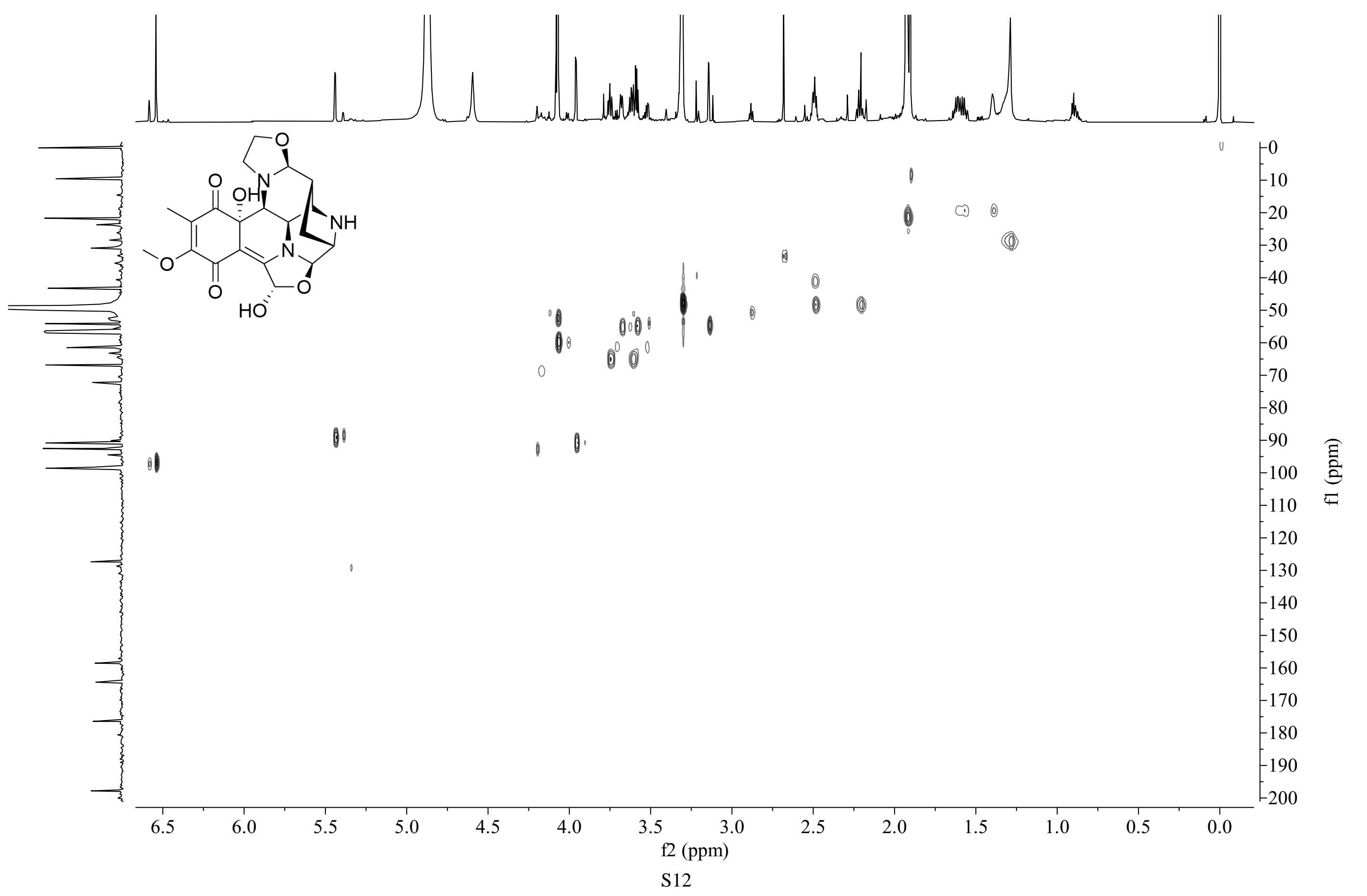


Figure S8. ${ }^{1} \mathrm{H}-{ }^{-1} \mathrm{H}$ COSY spectrum of 3 in $\mathrm{CD}_{3} \mathrm{OD}$

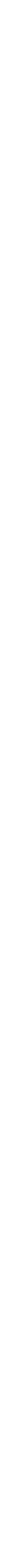


Figure S9. $\mathrm{HMBC}$ spectrum of 3 in $\mathrm{CD}_{3} \mathrm{OD}$

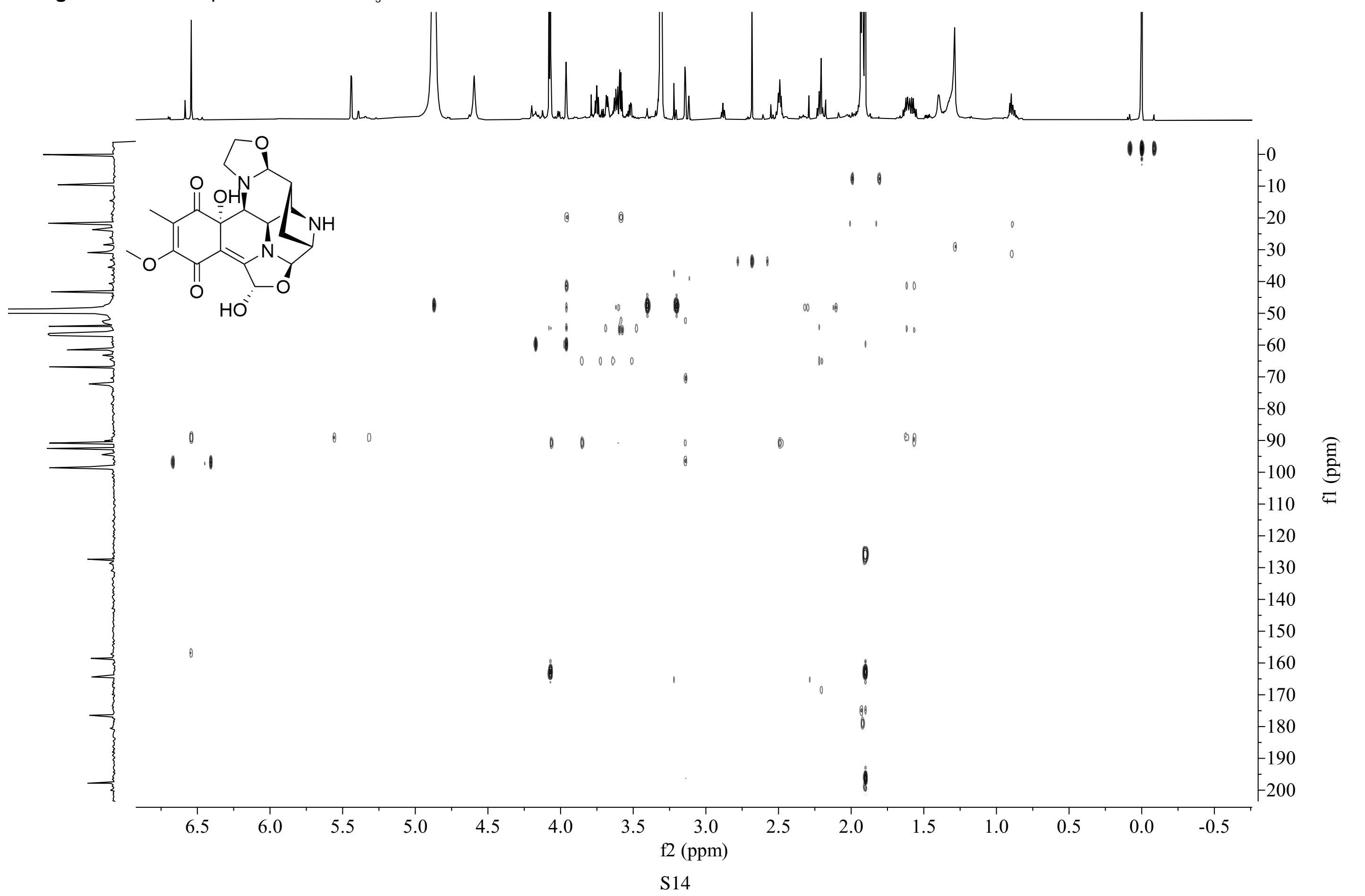


Figure S10. NOESY spectrum of 3 in $\mathrm{CD}_{3} \mathrm{OD}$

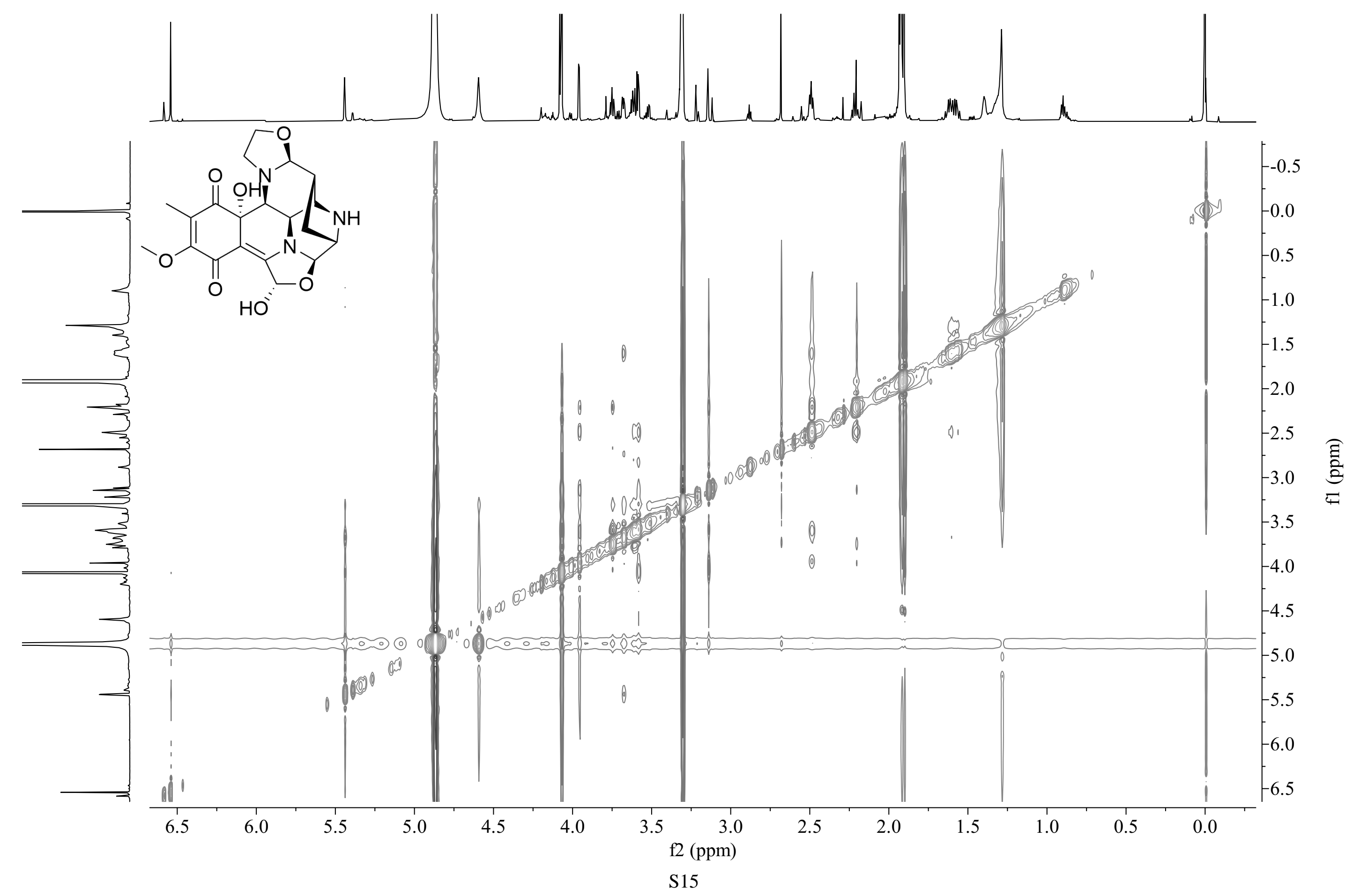


Figure S11. ${ }^{1} \mathrm{H}$ NMR $(700 \mathrm{MHz})$ spectrum of 4 in $\mathrm{CD}_{3} \mathrm{OD}$

అల

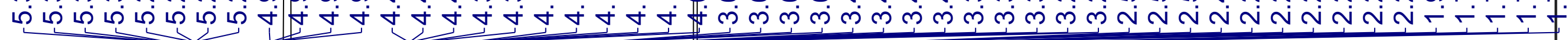

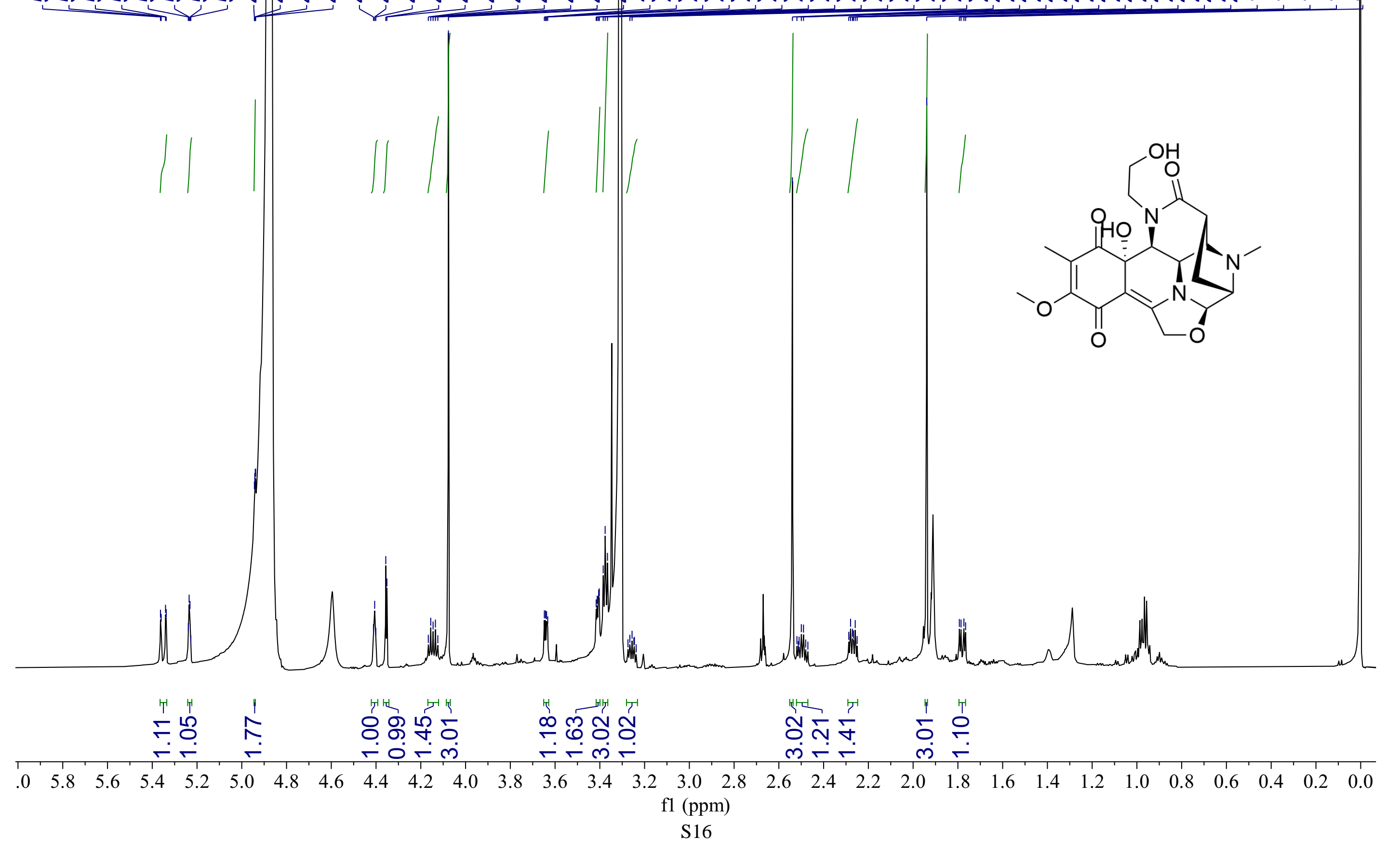


Figure S12. ${ }^{13} \mathrm{C}\left\{{ }^{1} \mathrm{H}\right\}$ NMR (175 MHz) spectrum of 4 in $\mathrm{CD}_{3} \mathrm{OD}$

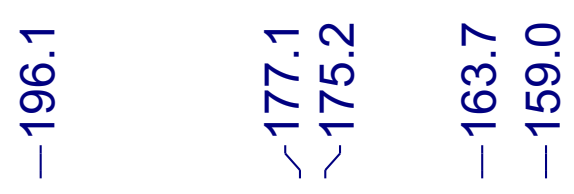

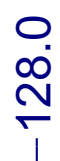

m.

ஸे ธั

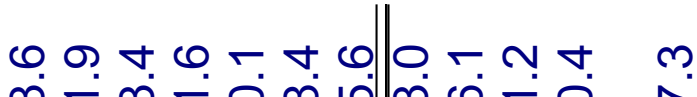

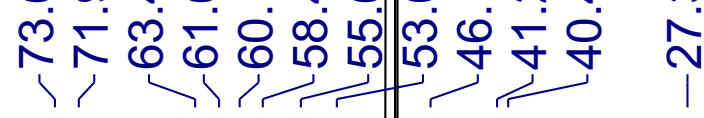
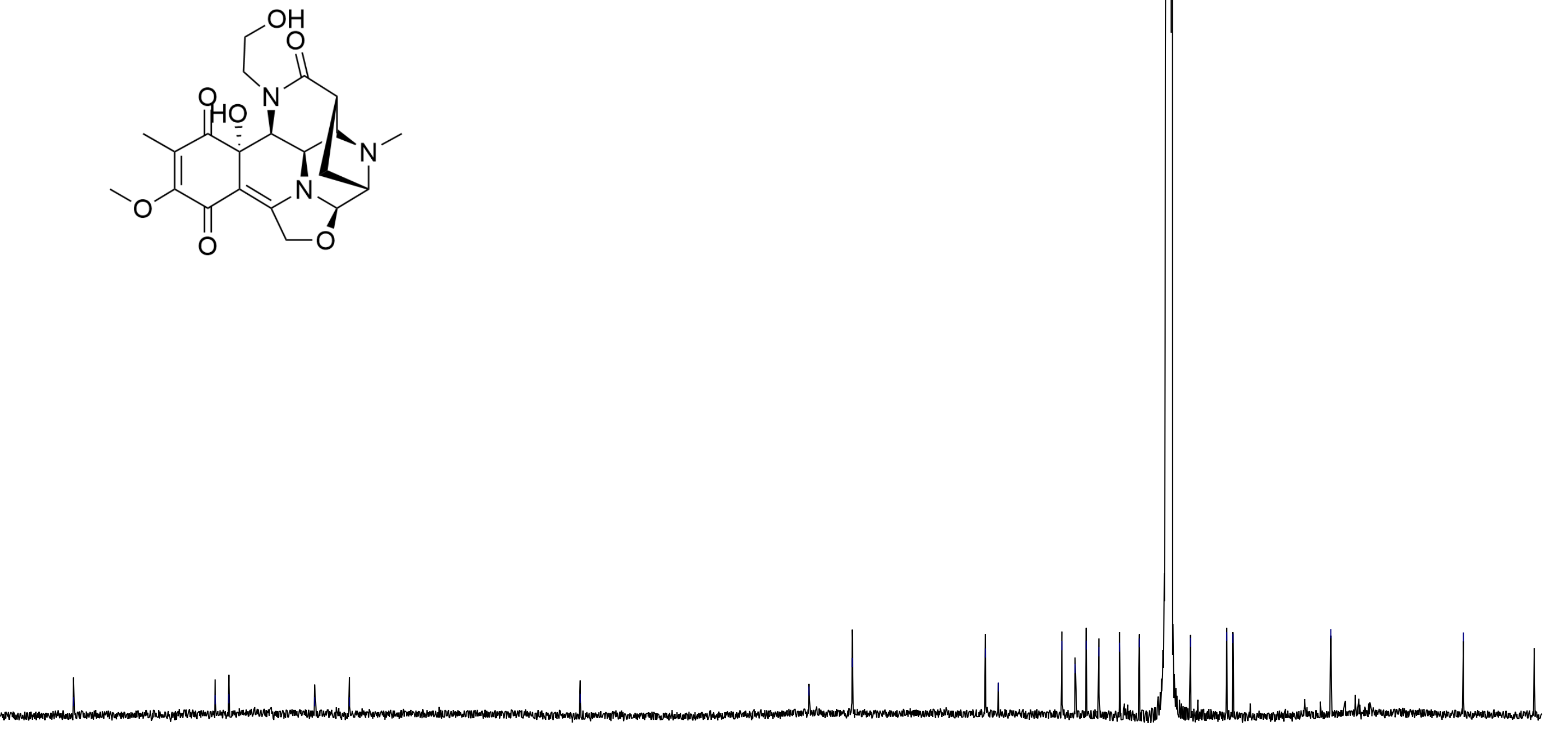
Figure S13. $\mathrm{HMQC}$ spectrum of 4 in $\mathrm{CD}_{3} \mathrm{OD}$

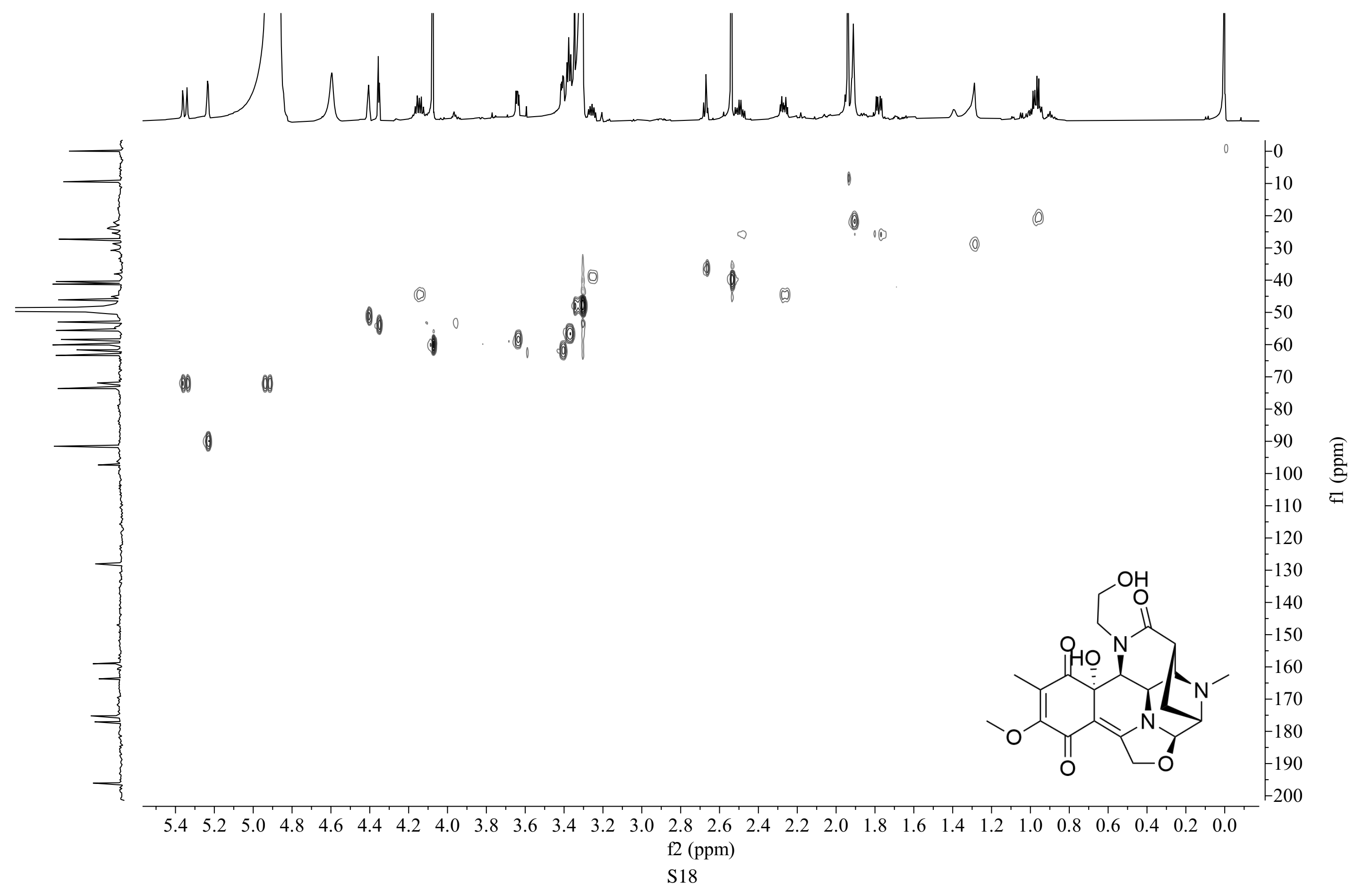


Figure S14. ${ }^{1} \mathrm{H}-{ }^{1} \mathrm{H}$ COSY spectrum of 4 in $\mathrm{CD}_{3} \mathrm{OD}$

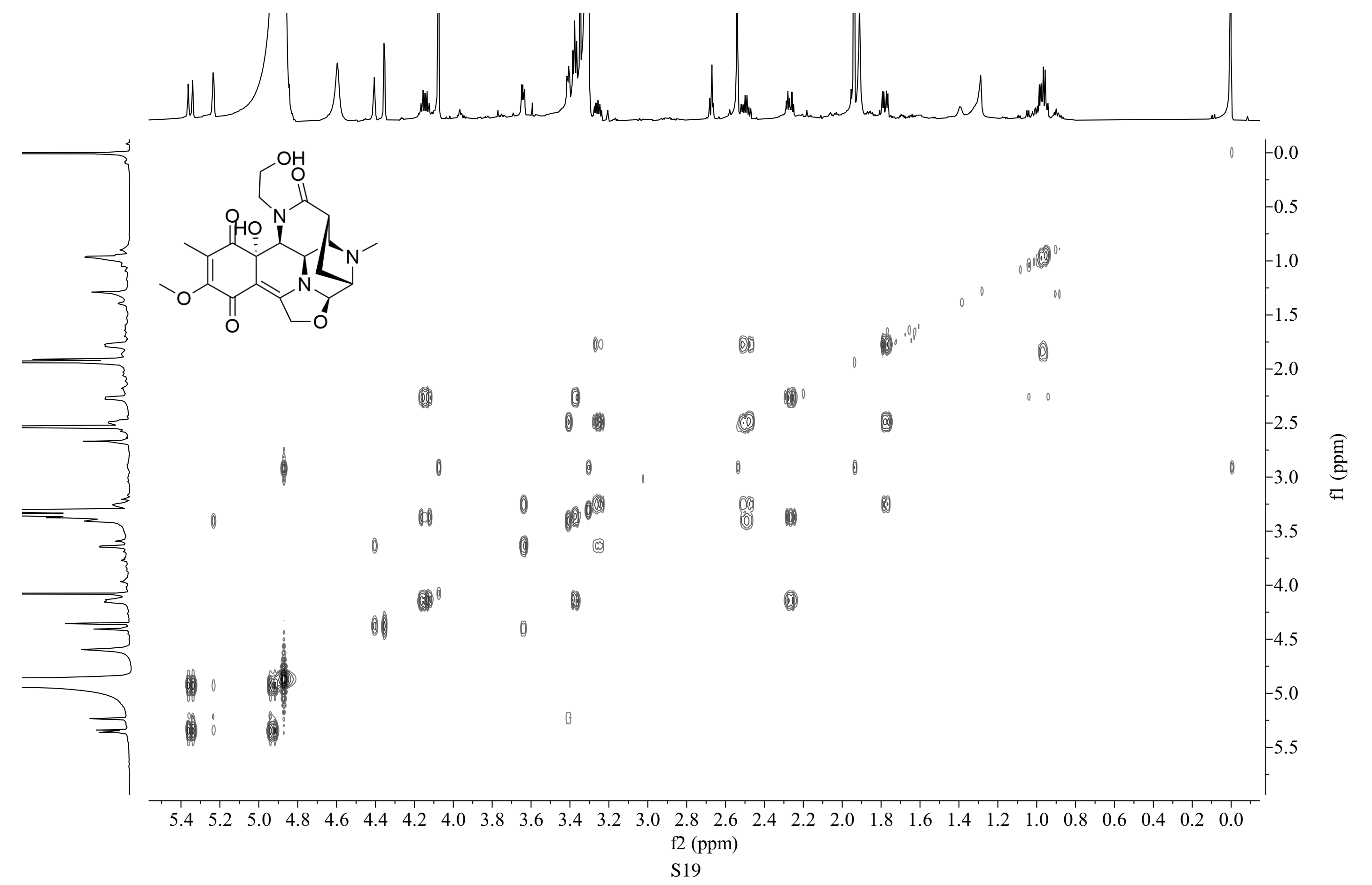


Figure S15. $\mathrm{HMBC}$ spectrum of 4 in $\mathrm{CD}_{3} \mathrm{OD}$
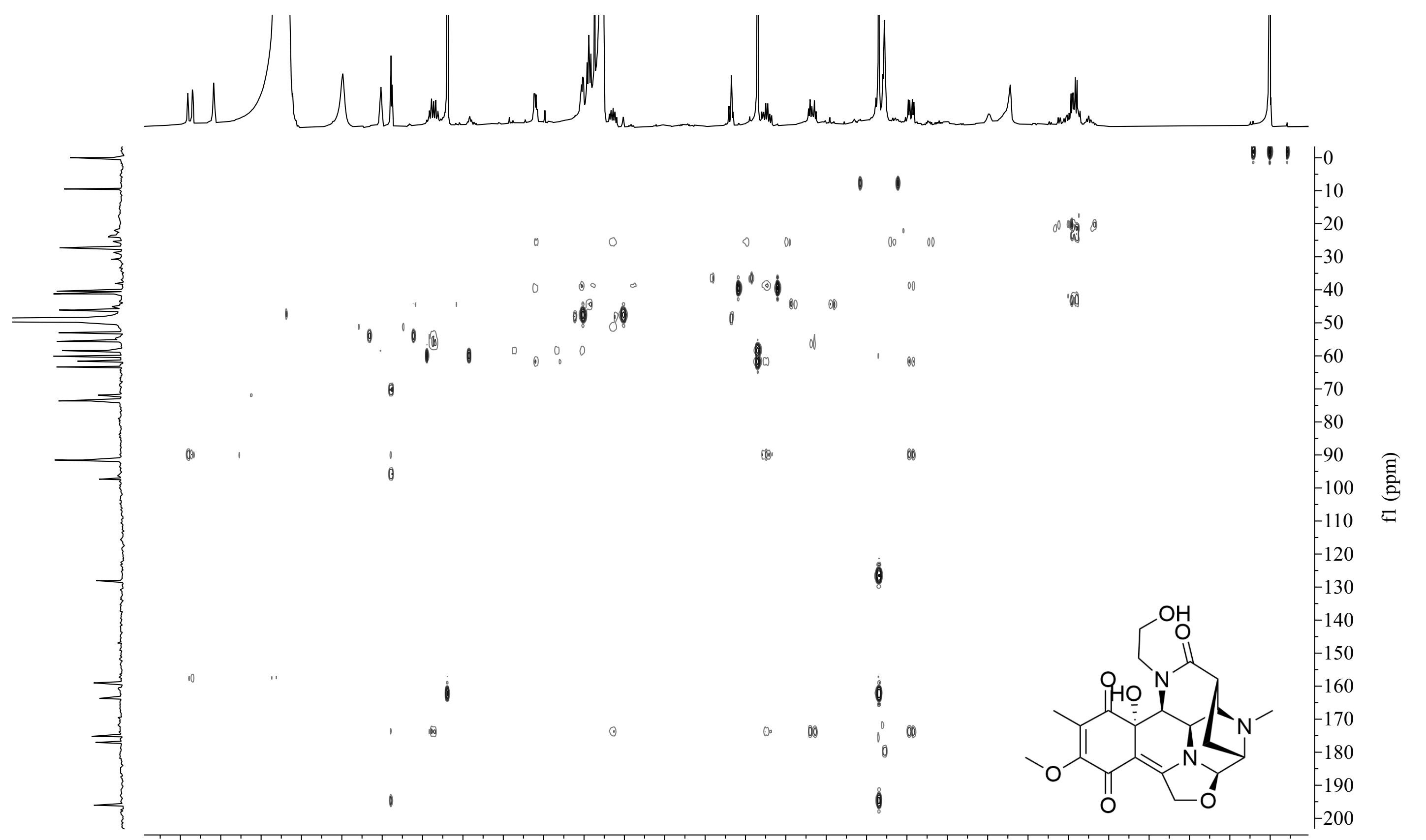

$\begin{array}{lllllllllllllllllllllllllllll}5.4 & 5.2 & 5.0 & 4.8 & 4.6 & 4.4 & 4.2 & 4.0 & 3.8 & 3.6 & 3.4 & 3.2 & 3.0 & 2.8 & 2.6 & 2.4 & 2.2 & 2.0 & 1.8 & 1.6 & 1.4 & 1.2 & 1.0 & 0.8 & 0.6 & 0.4 & 0.2 & 0.0\end{array}$ $\mathrm{f} 2(\mathrm{ppm})$ 
Figure S16. NOESY spectrum of 4 in $\mathrm{CD}_{3} \mathrm{OD}$

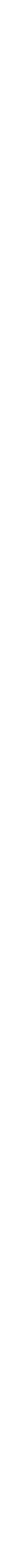


Figure S17. ${ }^{1} \mathrm{H}$ NMR $(700 \mathrm{MHz})$ spectrum of 5 in $\mathrm{CD}_{3} \mathrm{OD}$

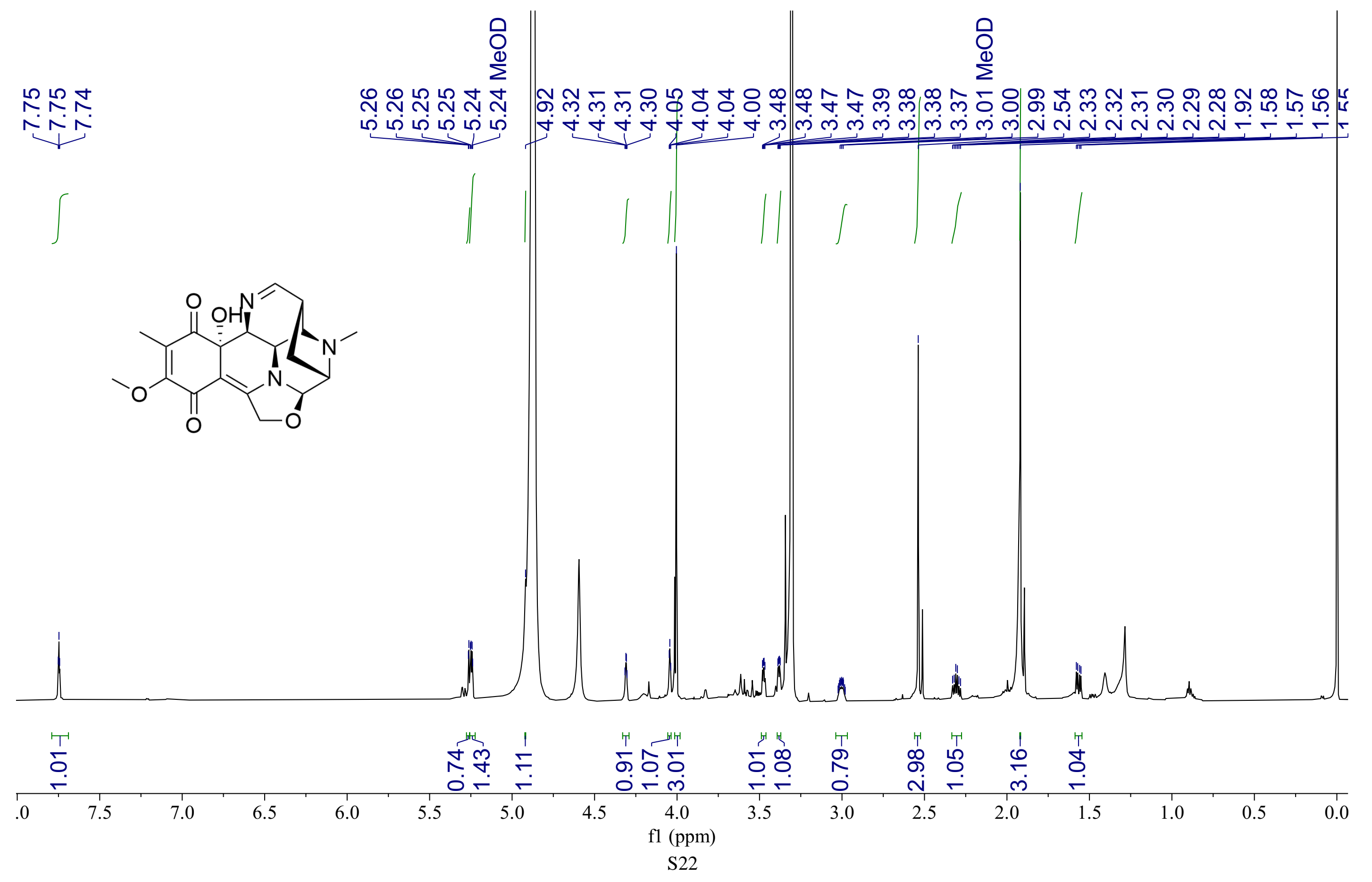


Figure S18. ${ }^{13} \mathrm{C}\left\{{ }^{1} \mathrm{H}\right\} \mathrm{NMR}(175 \mathrm{MHz})$ spectrum of 5 in $\mathrm{CD}_{3} \mathrm{OD}$

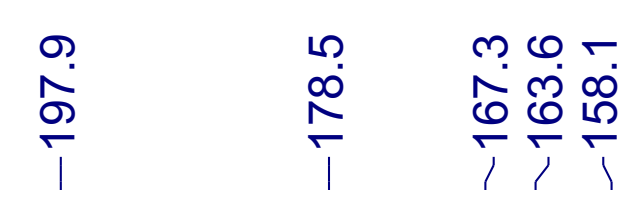

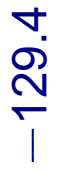

$\frac{10}{6}$

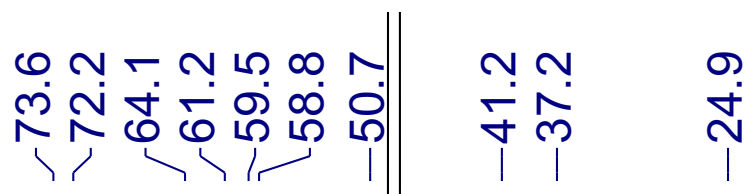

L

i

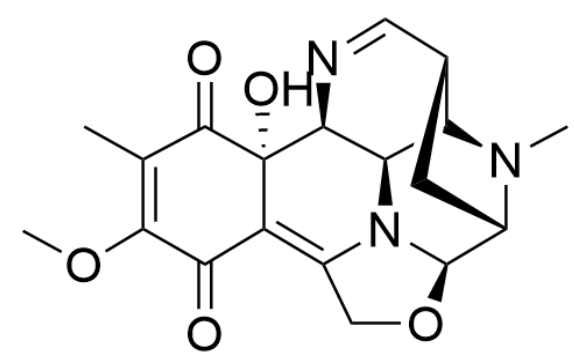

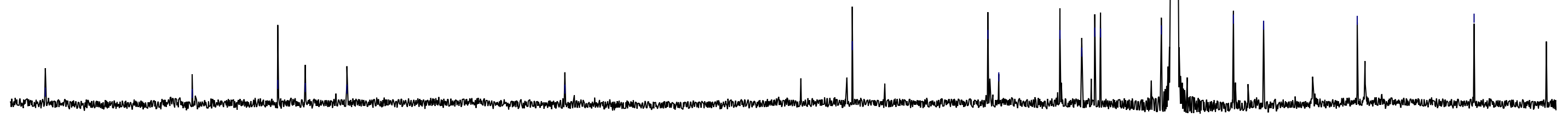


Figure S19. $\mathrm{HMQC}$ spectrum of 5 in $\mathrm{CD}_{3} \mathrm{OD}$
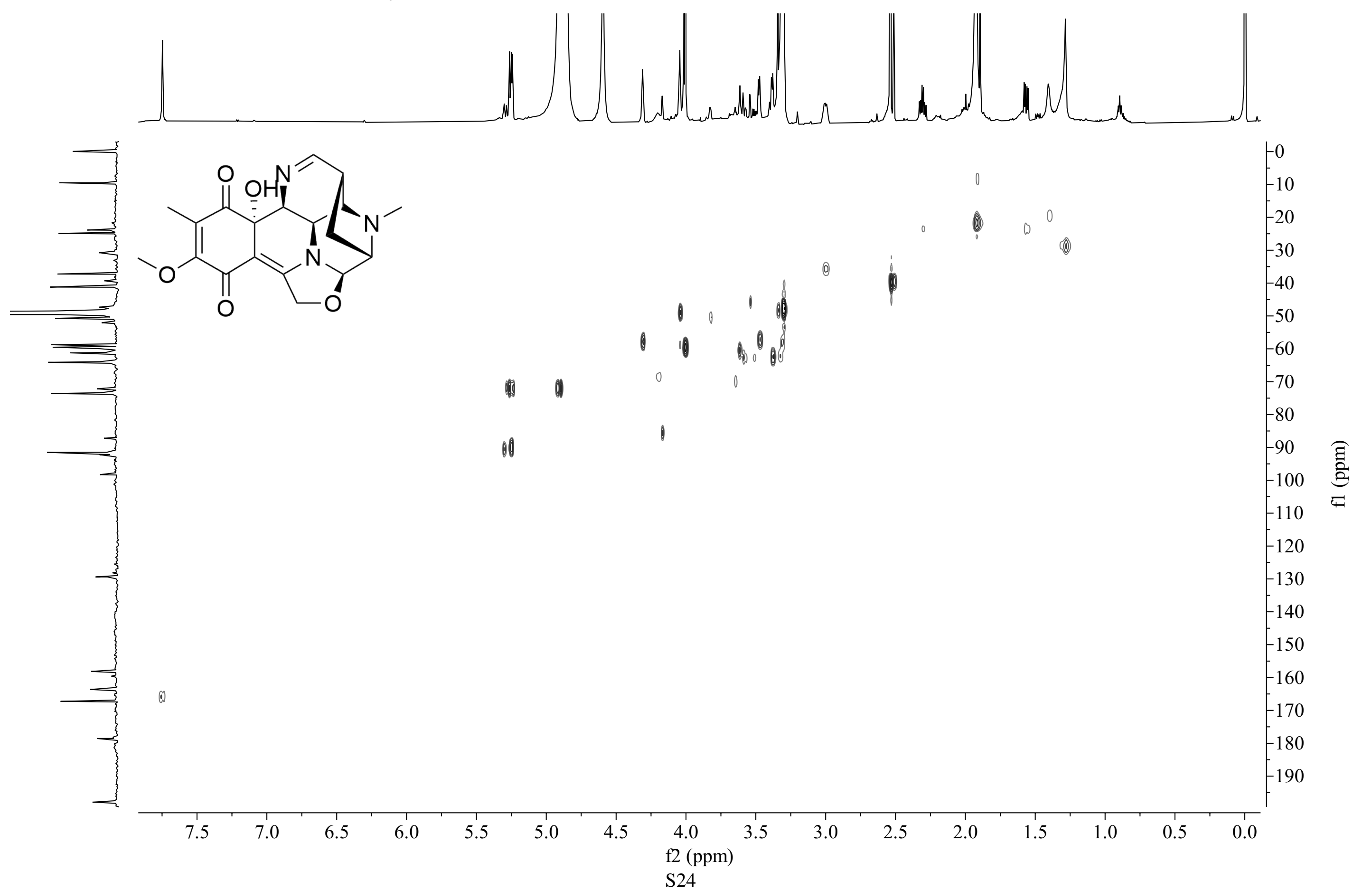
Figure S20. ${ }^{1} \mathrm{H}-{ }^{-} \mathrm{H}$ COSY spectrum of $\mathbf{5}$ in $\mathrm{CD}_{3} \mathrm{OD}$

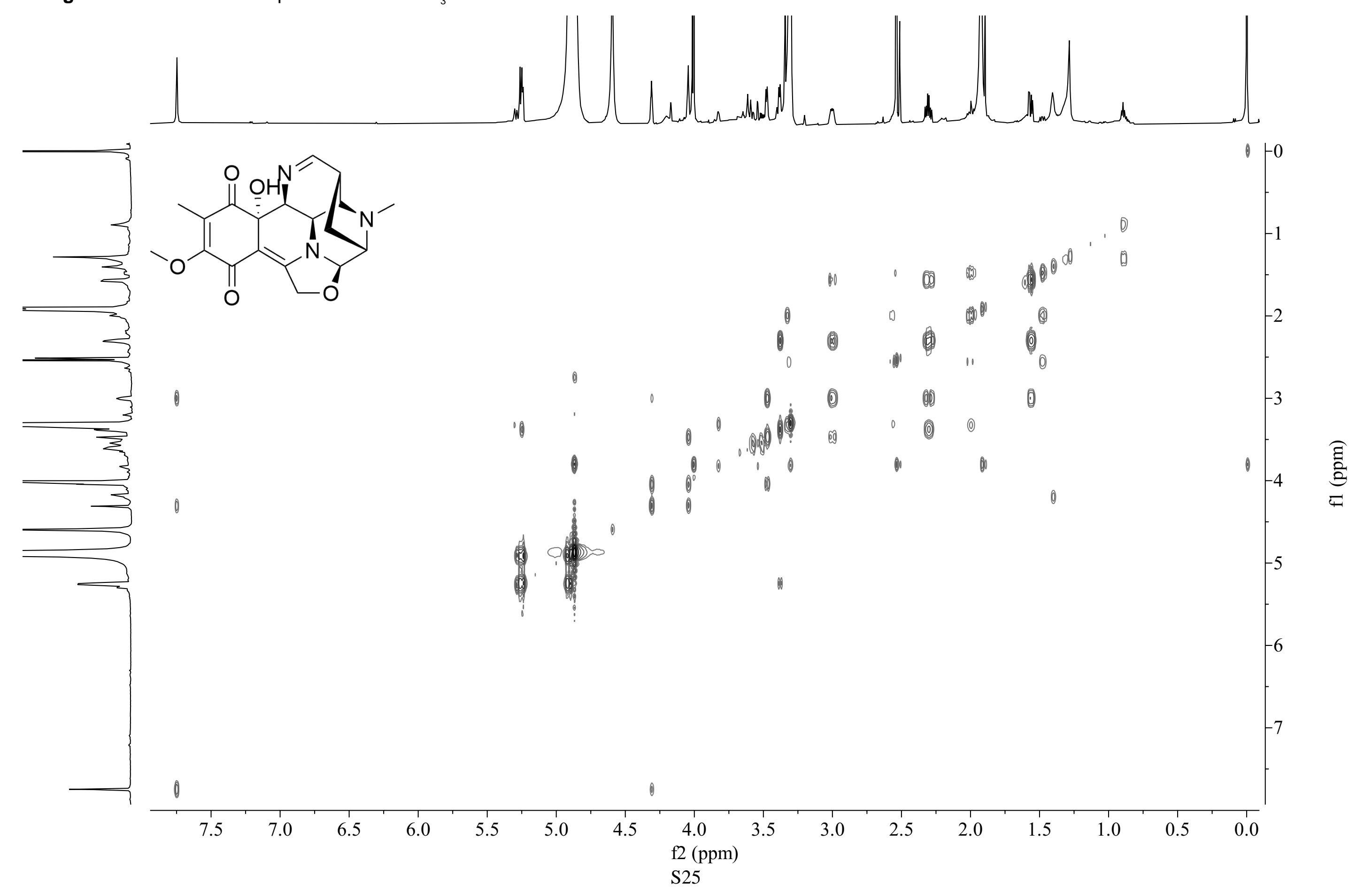


Figure S21. $\mathrm{HMBC}$ spectrum of $\mathbf{5}$ in $\mathrm{CD}_{3} \mathrm{OD}$

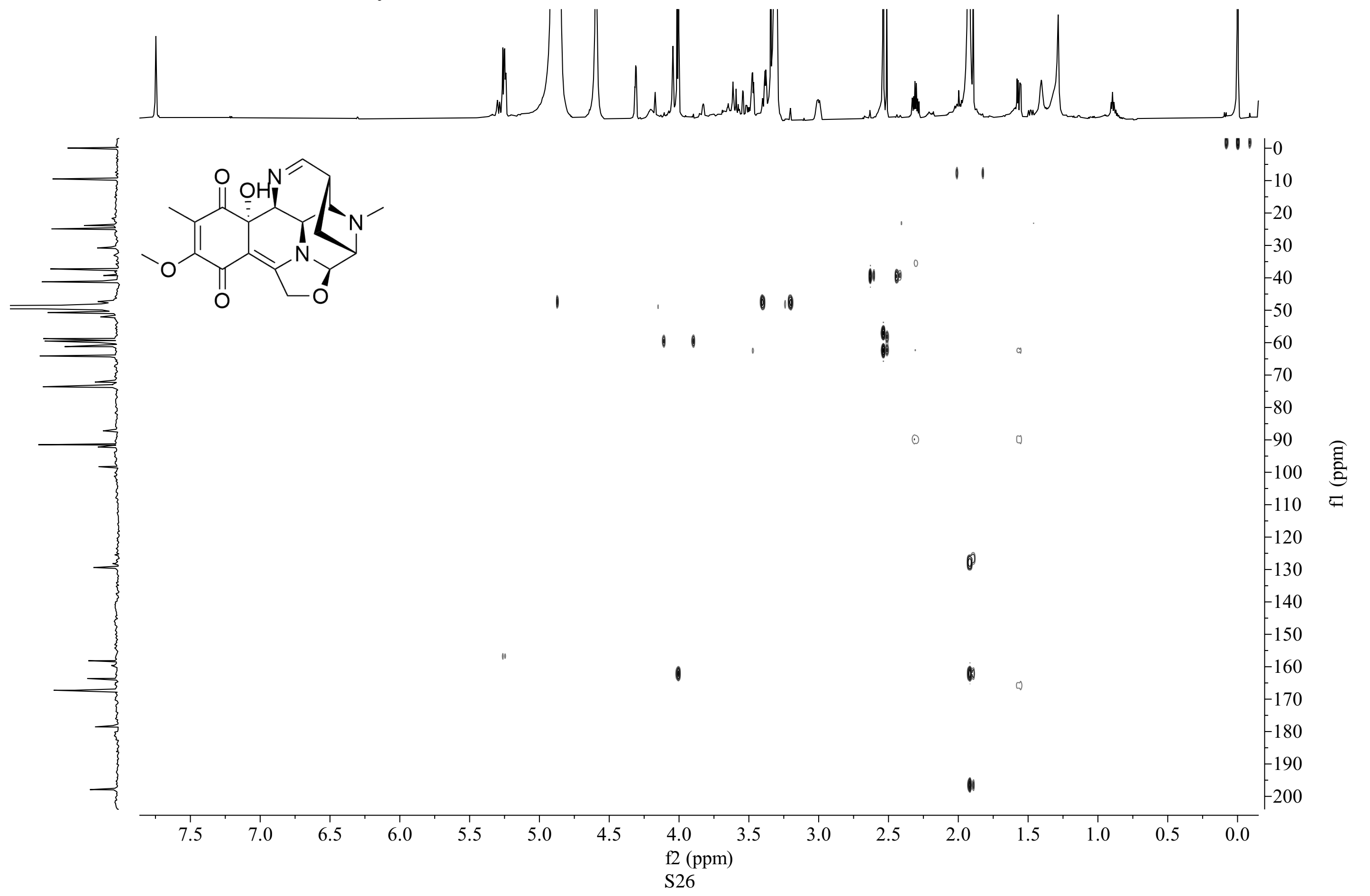


Figure S22. NOESY spectrum of 5 in $\mathrm{CD}_{3} \mathrm{OD}$

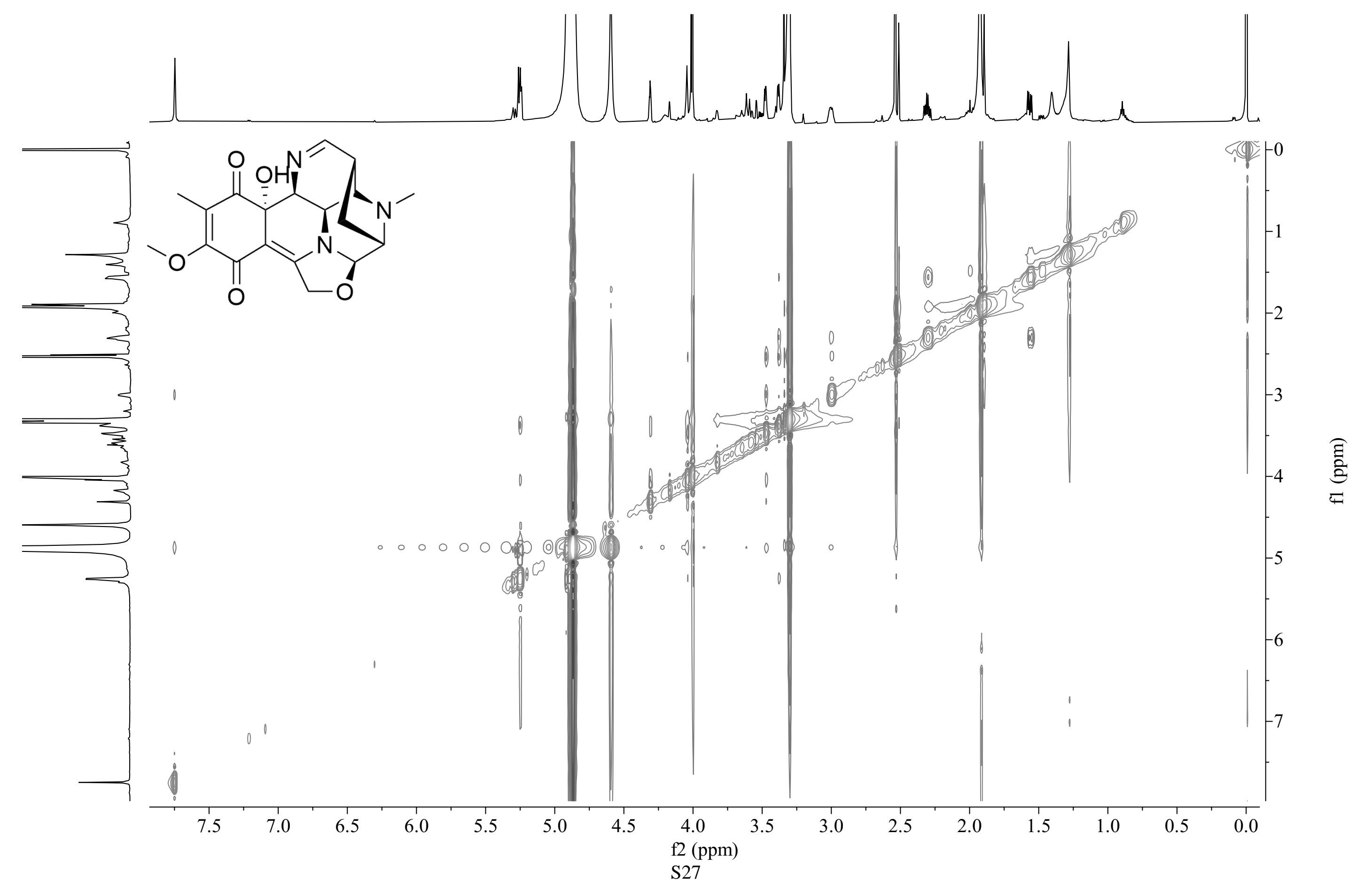


Figure S23. ${ }^{1} \mathrm{H}$ NMR $\left(700 \mathrm{MHz}\right.$ ) spectrum of 6 in $\mathrm{CD}_{3} \mathrm{OD}$

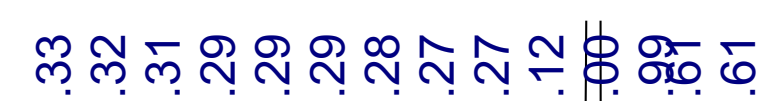

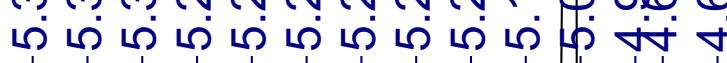

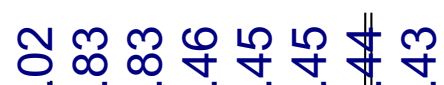
पे लं mं लं

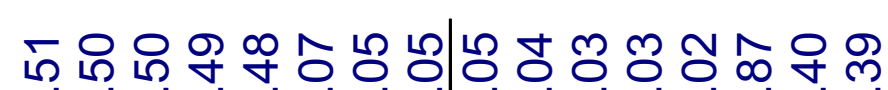

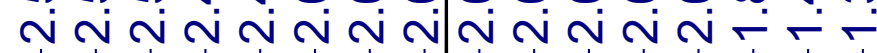

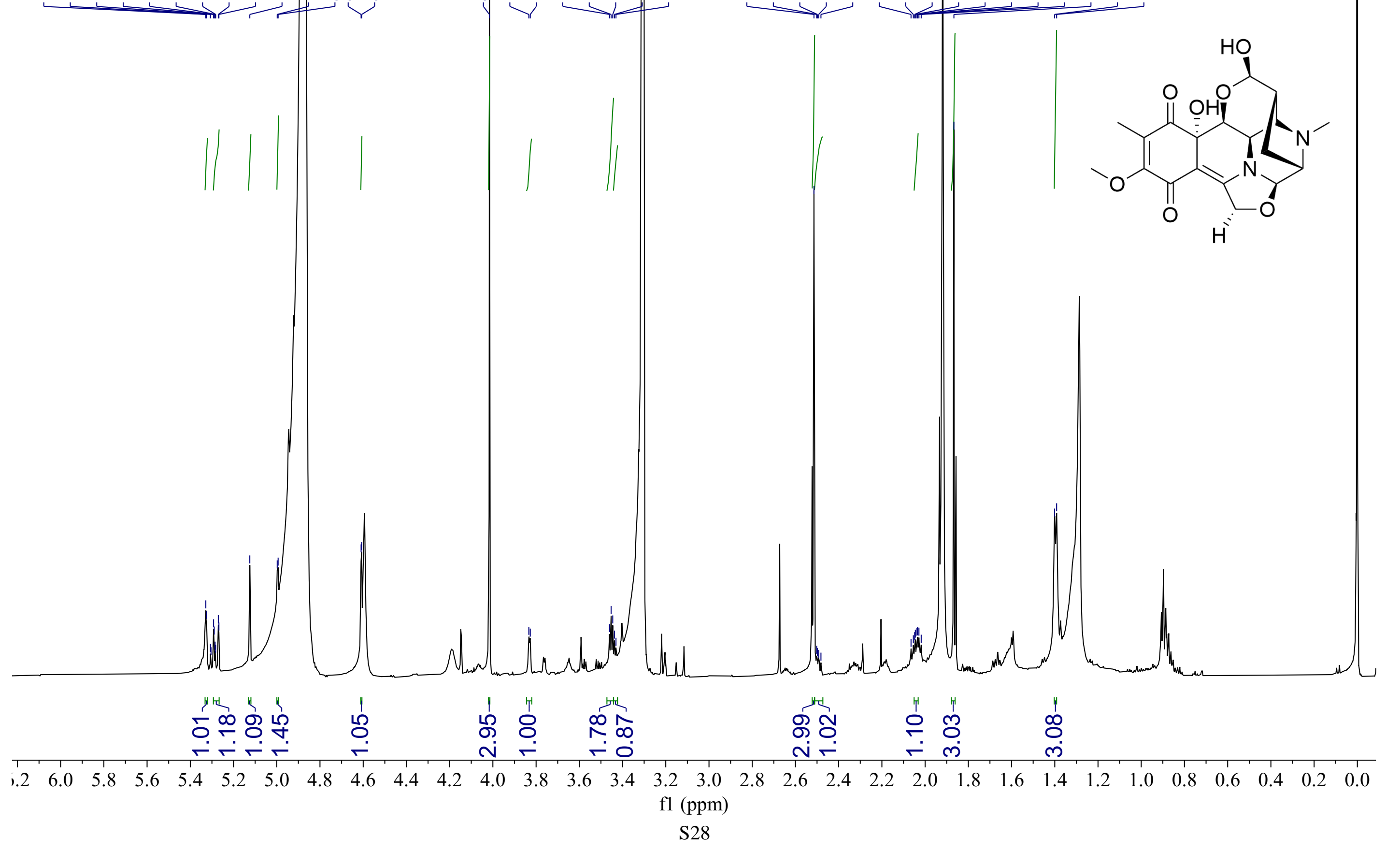


Figure S24. ${ }^{13} \mathrm{C}\left\{{ }^{1} \mathrm{H}\right\}$ NMR (175 MHz) spectrum of 6 in $\mathrm{CD}_{3} \mathrm{OD}$

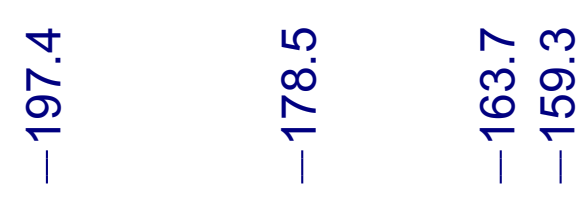

$\stackrel{m}{\stackrel{\infty}{N}}$

$\forall \infty \circ$

$\infty$ mं

क्रा

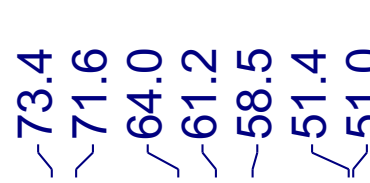

$\infty \infty \infty$

广 गे ते

$\stackrel{+}{i}$

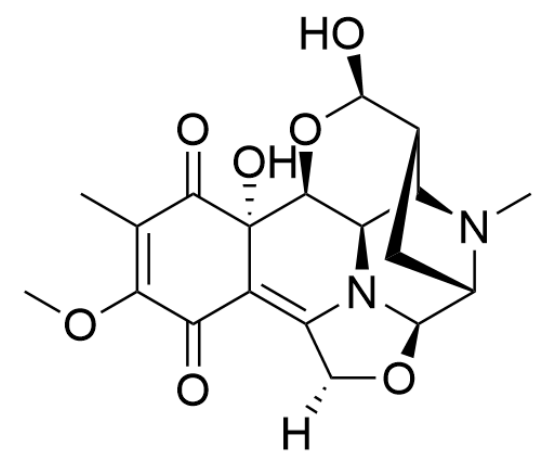

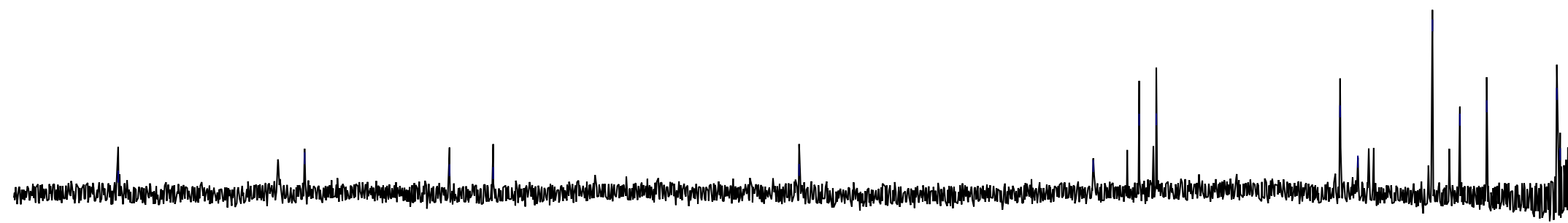




\section{Figure S25. $\mathrm{HMQC}$ spectrum of 6 in $\mathrm{CD}_{3} \mathrm{OD}$}

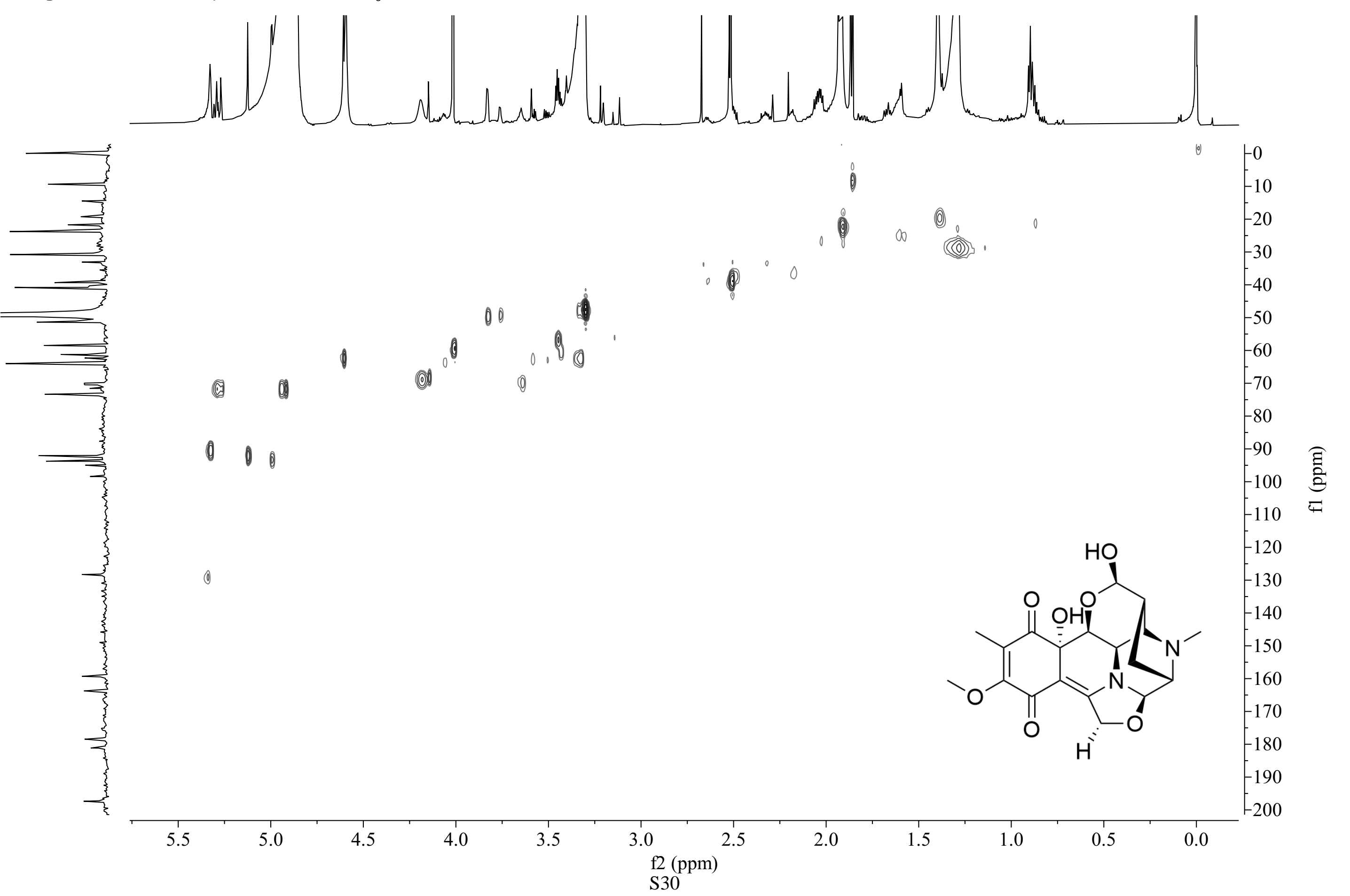


Figure S26. ' $\mathrm{H}-{ }^{-} \mathrm{H}$ COSY spectrum of 6 in $\mathrm{CD}_{3} \mathrm{OD}$

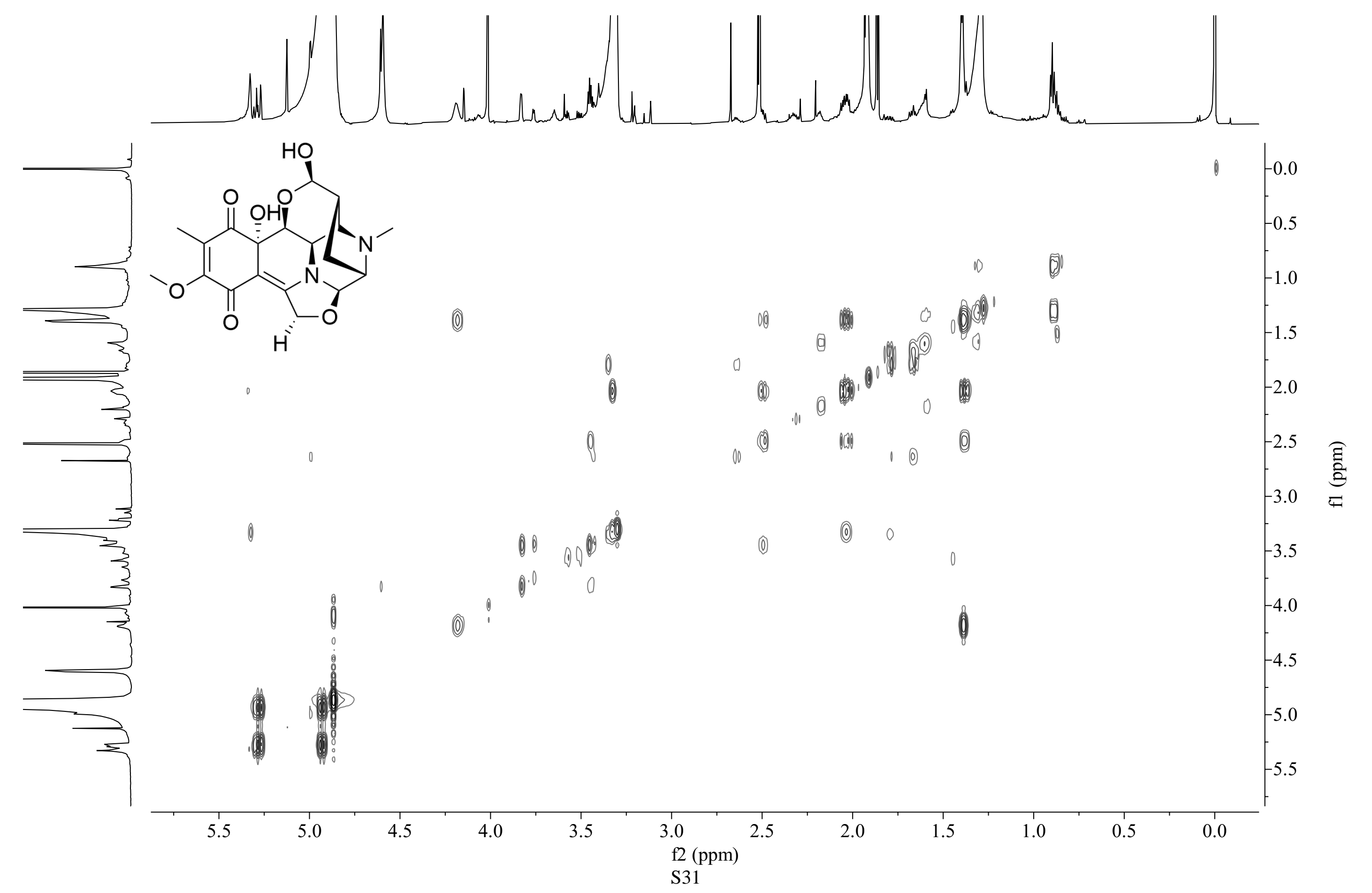


Figure S27. $\mathrm{HMBC}$ spectrum of $\mathbf{6}$ in $\mathrm{CD}_{3} \mathrm{OD}$

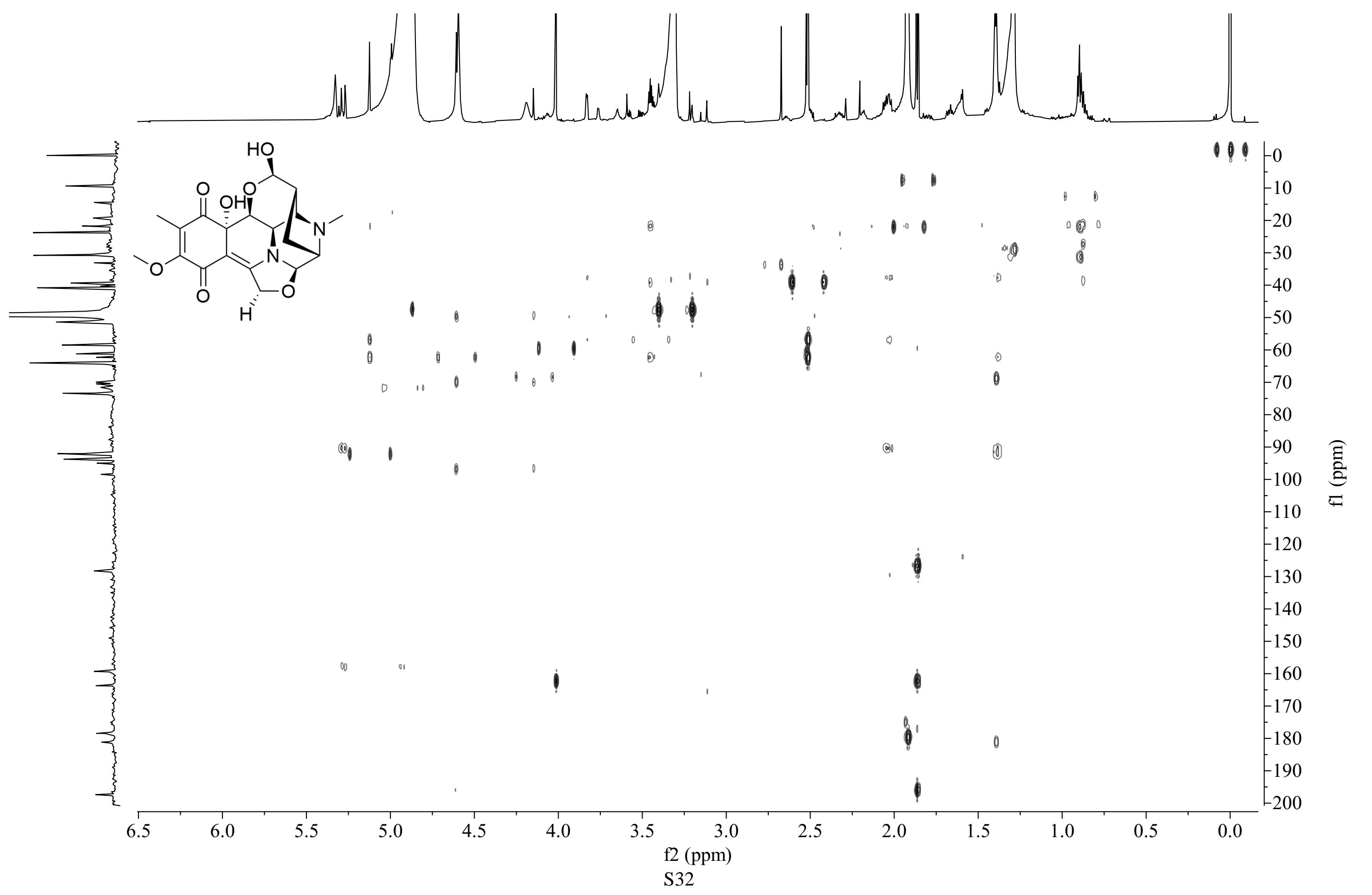


Figure S28. NOESY spectrum of 6 in $\mathrm{CD}_{3} \mathrm{OD}$

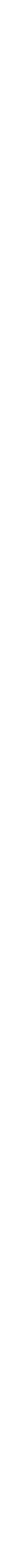


Figure S29. ${ }^{1} \mathrm{H}$ NMR $(700 \mathrm{MHz})$ spectrum of 7 in $\mathrm{CD}_{3} \mathrm{OD}$

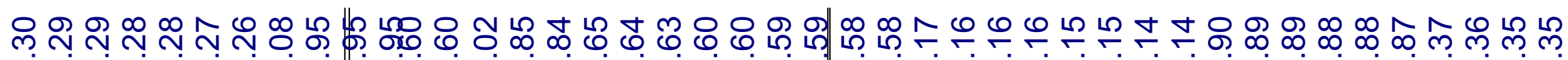

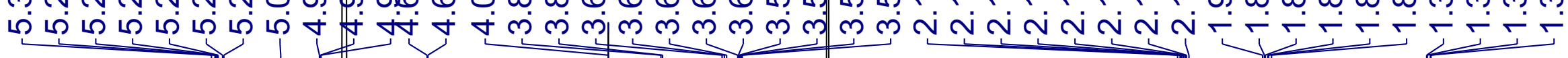

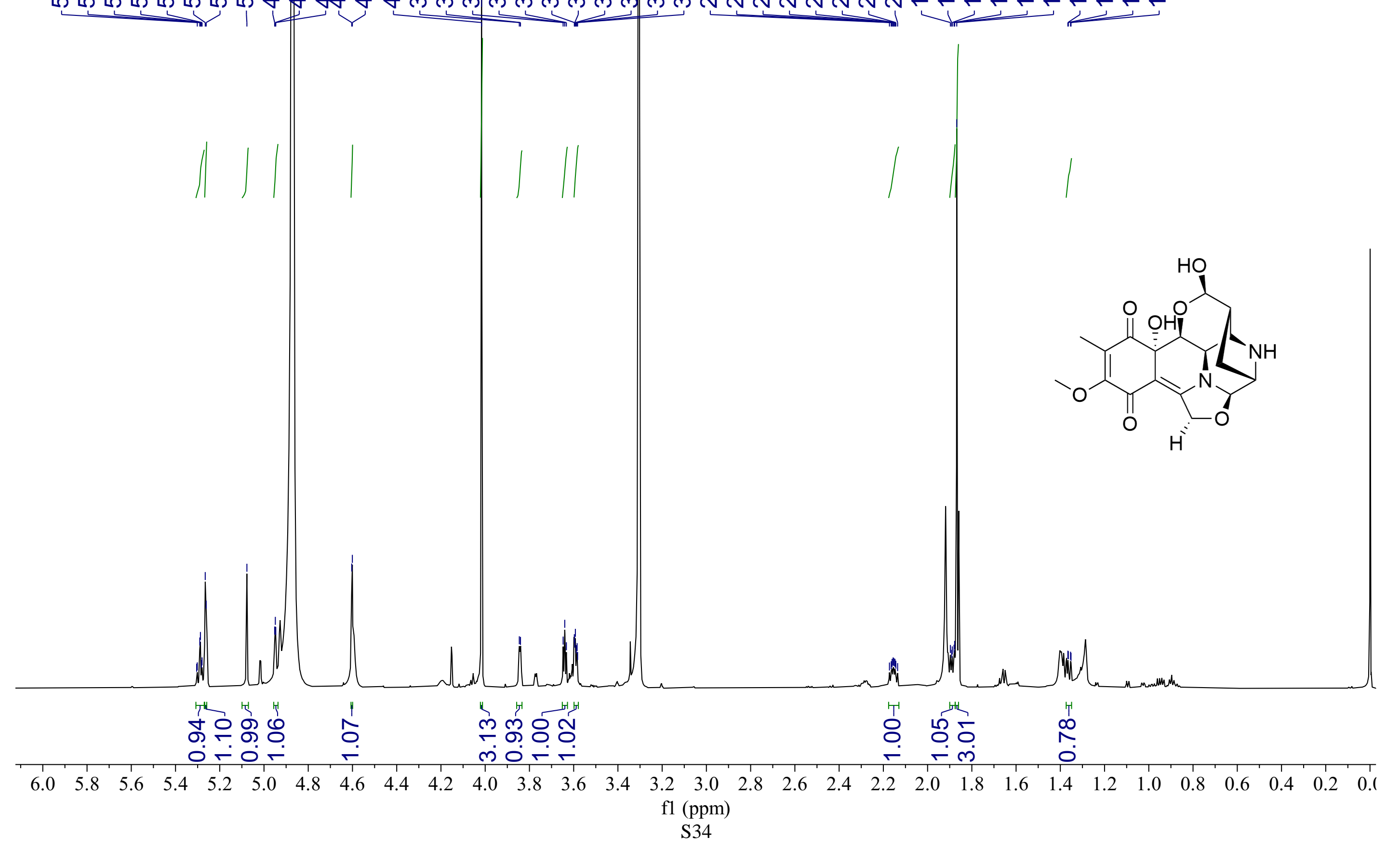


Figure S30. ${ }^{13} \mathrm{C}\left\{{ }^{1} \mathrm{H}\right\} \mathrm{NMR}(175 \mathrm{MHz})$ spectrum of 7 in $\mathrm{CD}_{3} \mathrm{OD}$

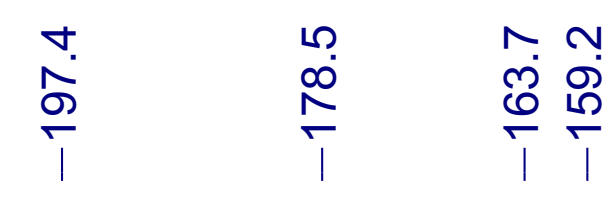

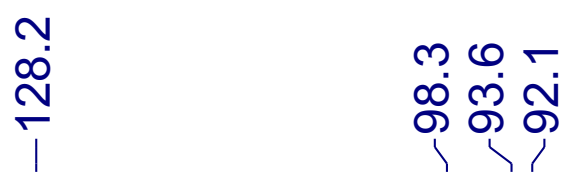

$m$

ल

ก)

$\stackrel{+}{1}$

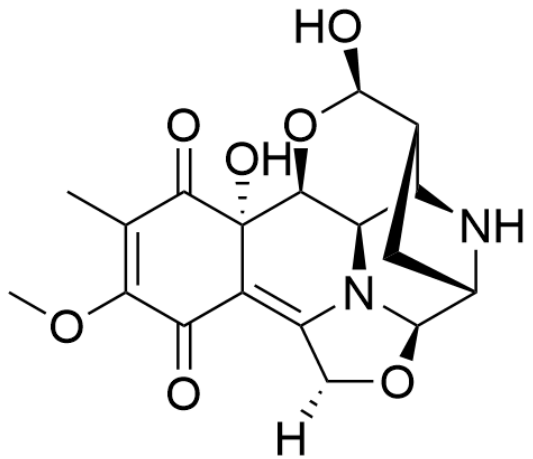


Figure S31. HMQC spectrum of 7 in $\mathrm{CD}_{3} \mathrm{OD}$

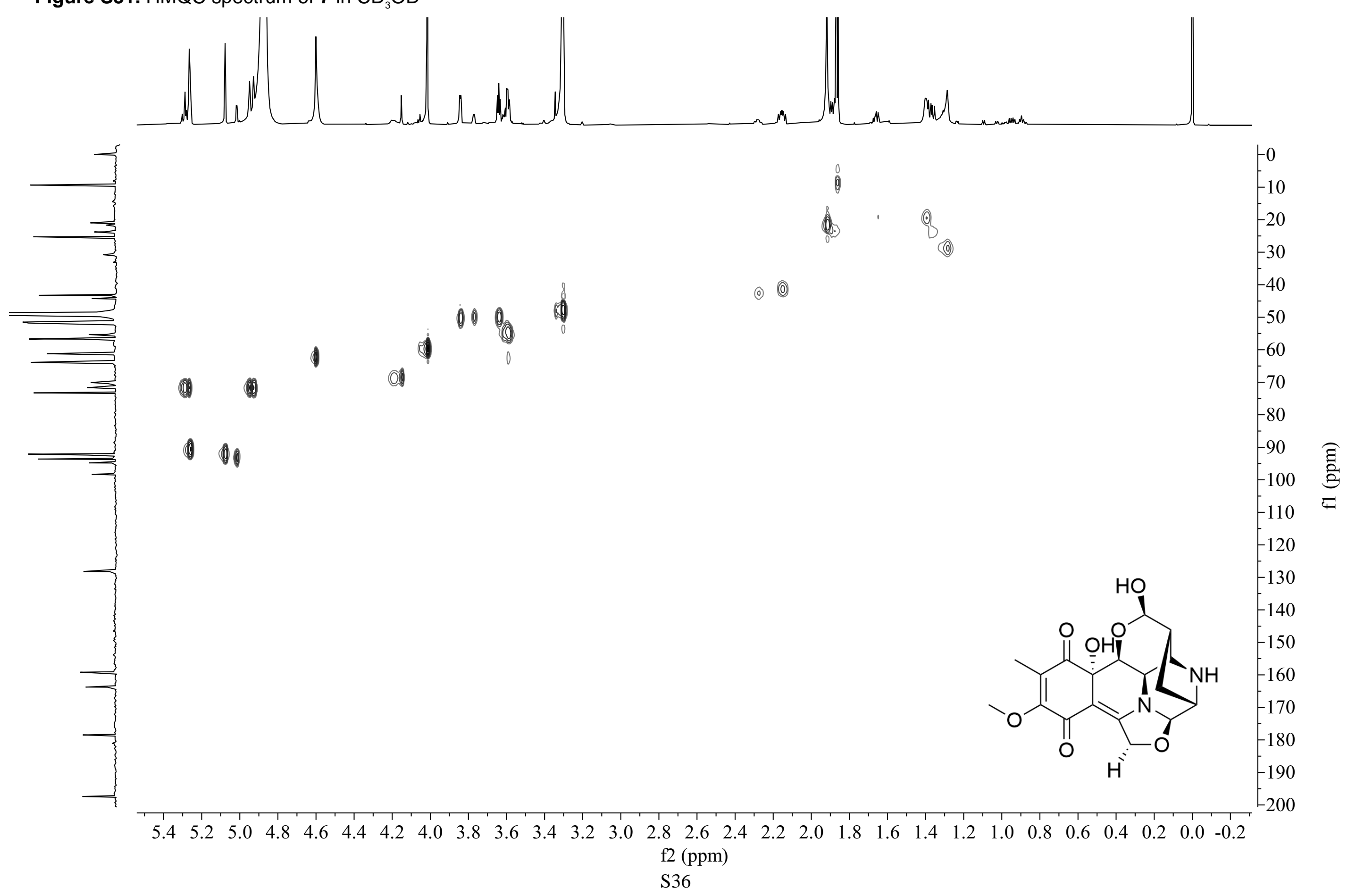


Figure S32. ${ }^{1} \mathrm{H}-{ }^{1} \mathrm{H}$ COSY spectrum of 7 in $\mathrm{CD}_{3} \mathrm{OD}$

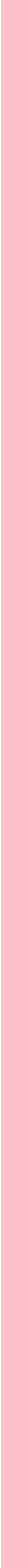


Figure S33. $\mathrm{HMBC}$ spectrum of $\mathbf{7}$ in $\mathrm{CD}_{3} \mathrm{OD}$

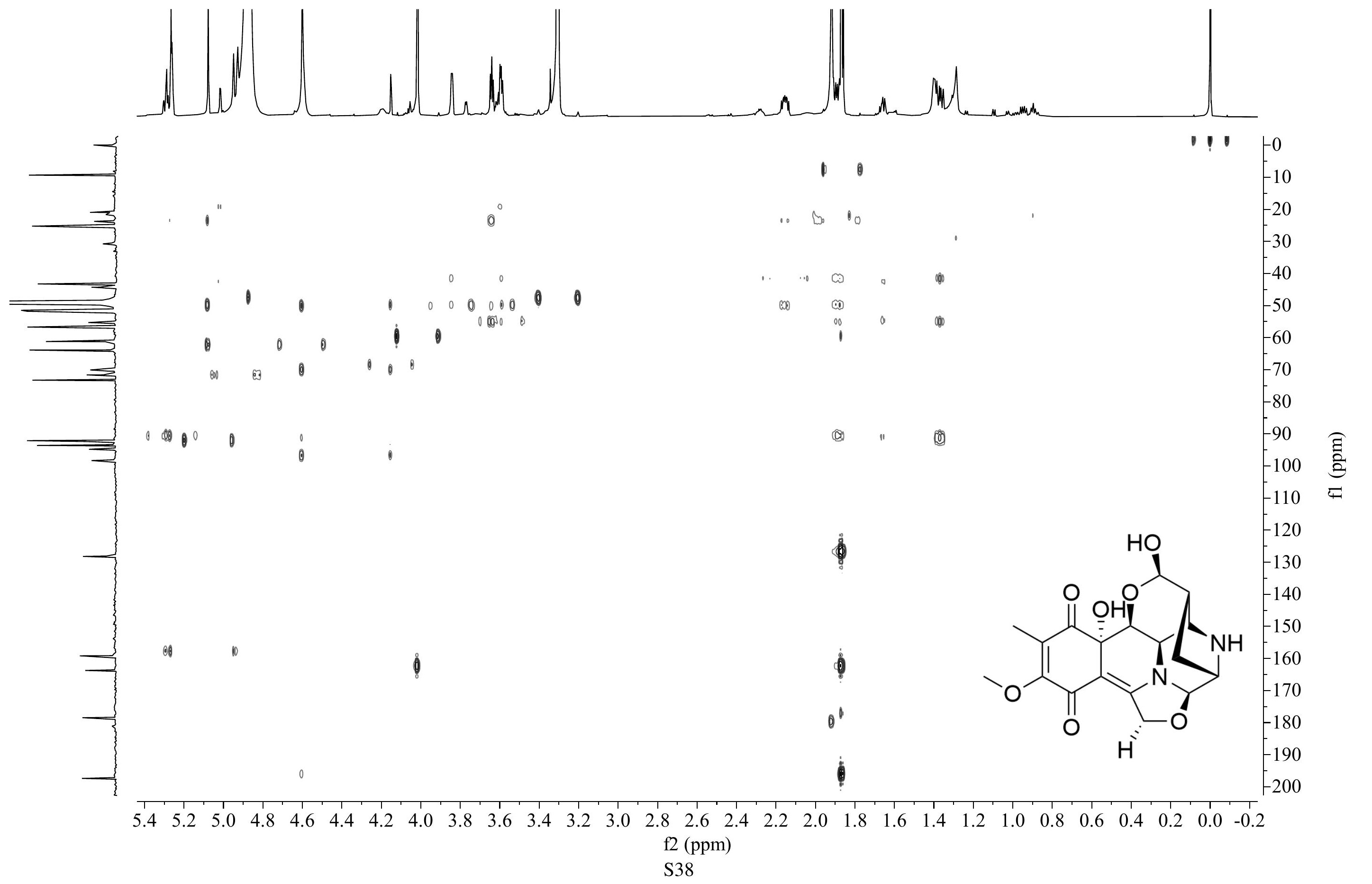


Figure S34. NOESY spectrum of 7 in $\mathrm{CD}_{3} \mathrm{OD}$

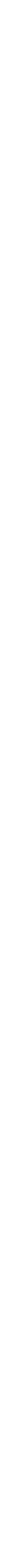


Figure S35. ${ }^{1} \mathrm{H}$ NMR $(700 \mathrm{MHz})$ spectrum of 8 in $\mathrm{CD}_{3} \mathrm{OD}$

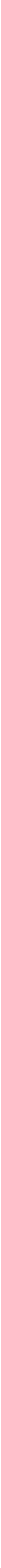


Figure S36. ${ }^{13} \mathrm{C}\left\{{ }^{1} \mathrm{H}\right\}$ NMR (175 MHz) spectrum of 8 in $\mathrm{CD}_{3} \mathrm{OD}$

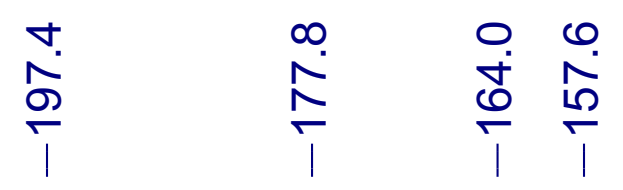
$\frac{\pi}{+\infty}$
N
の

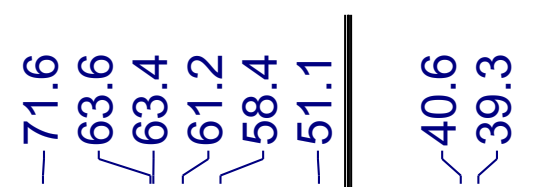
๗ָ

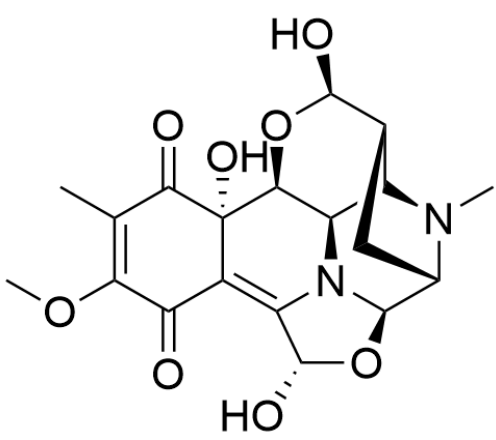


Figure S37. $\mathrm{HMQC}$ spectrum of 8 in $\mathrm{CD}_{3} \mathrm{OD}$

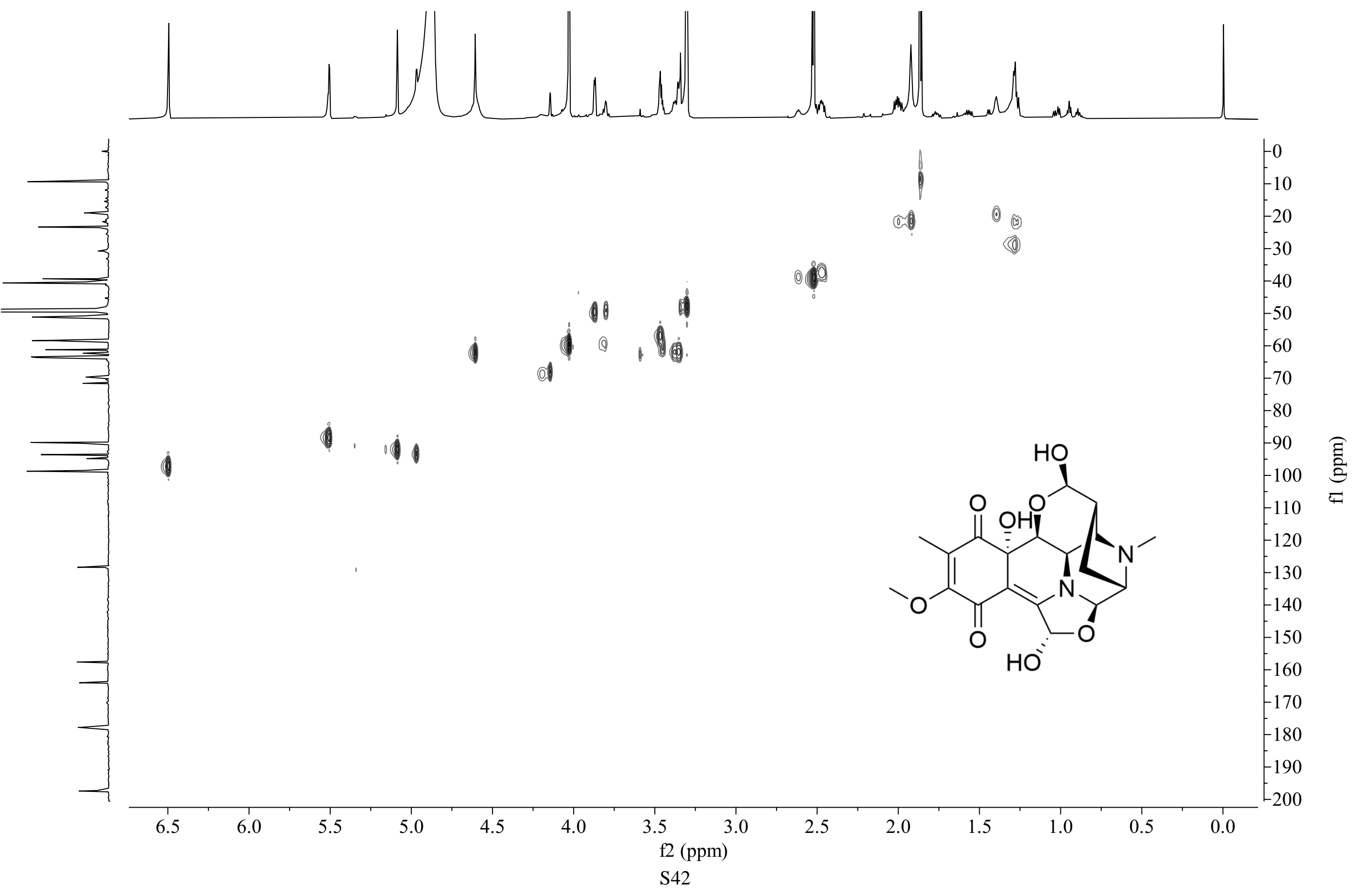


Figure S38. ${ }^{1} \mathrm{H}-{ }^{-1} \mathrm{H}$ COSY spectrum of 8 in $\mathrm{CD}_{3} \mathrm{OD}$

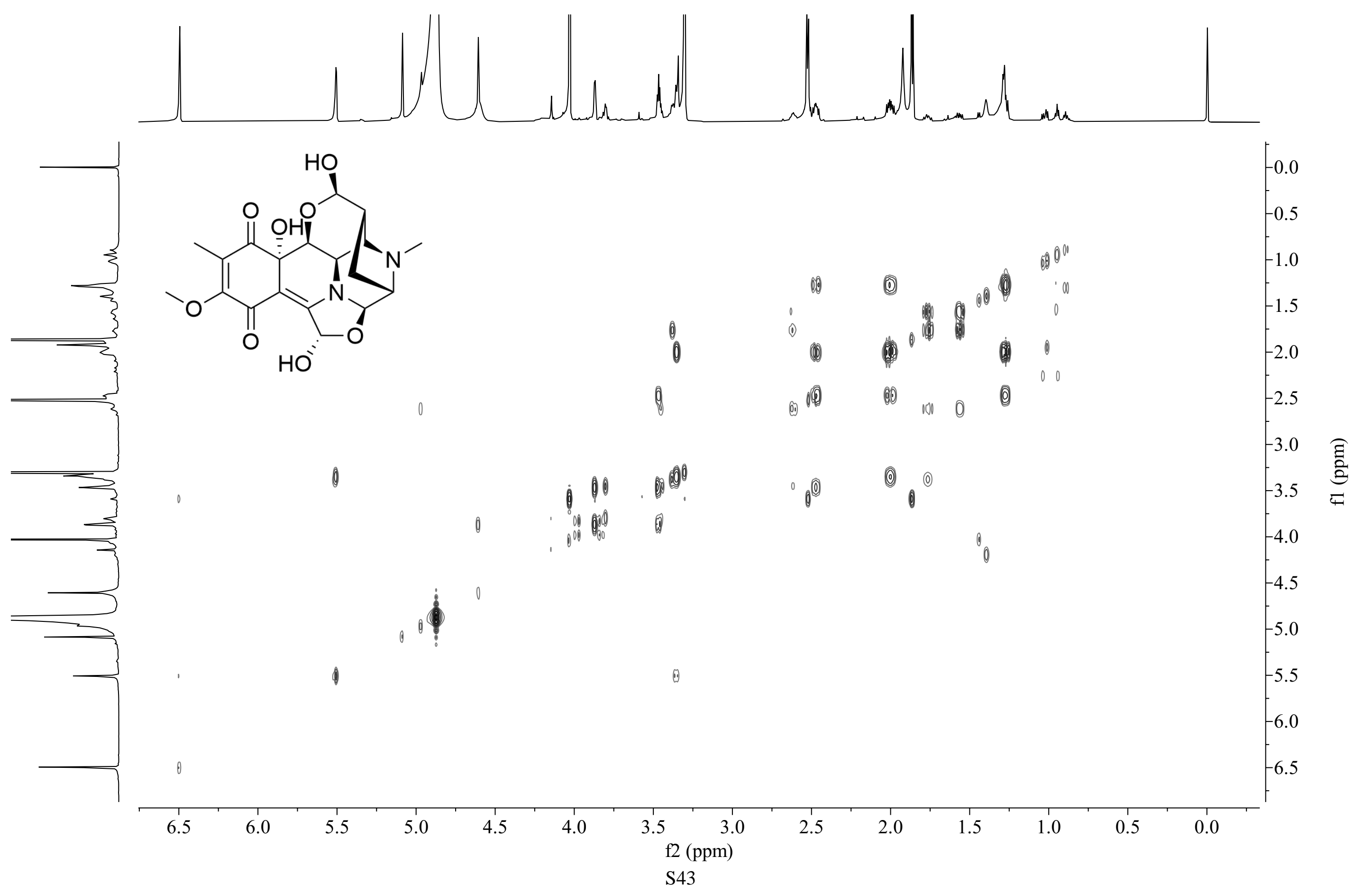


Figure S39. $\mathrm{HMBC}$ spectrum of 8 in $\mathrm{CD}_{3} \mathrm{OD}$

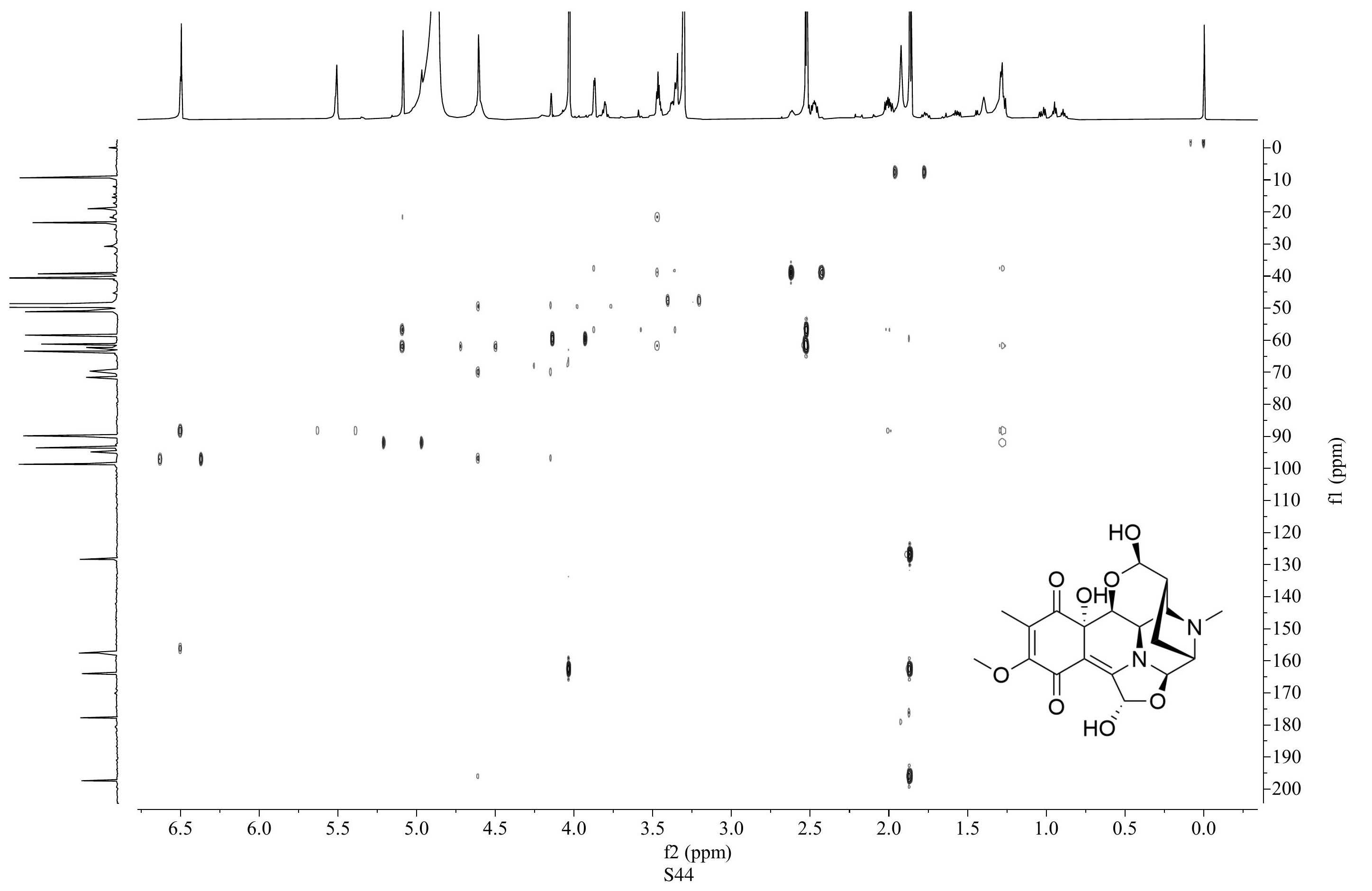


Figure S40. NOESY spectrum of 8 in $\mathrm{CD}_{3} \mathrm{OD}$

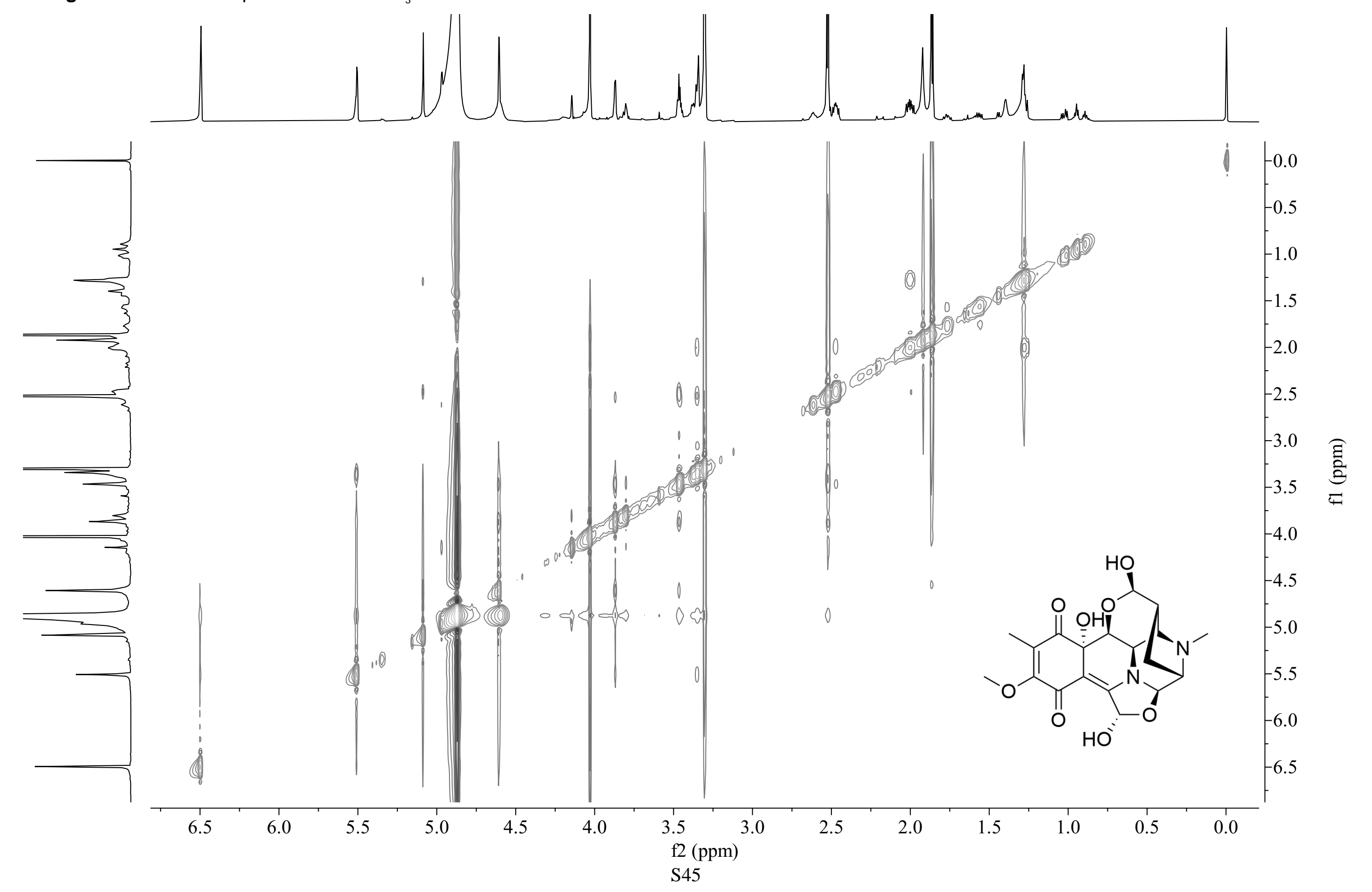


Figure S41. ${ }^{1} \mathrm{H}$ NMR $(700 \mathrm{MHz})$ spectrum of 9 in $\mathrm{CD}_{3} \mathrm{OD}$

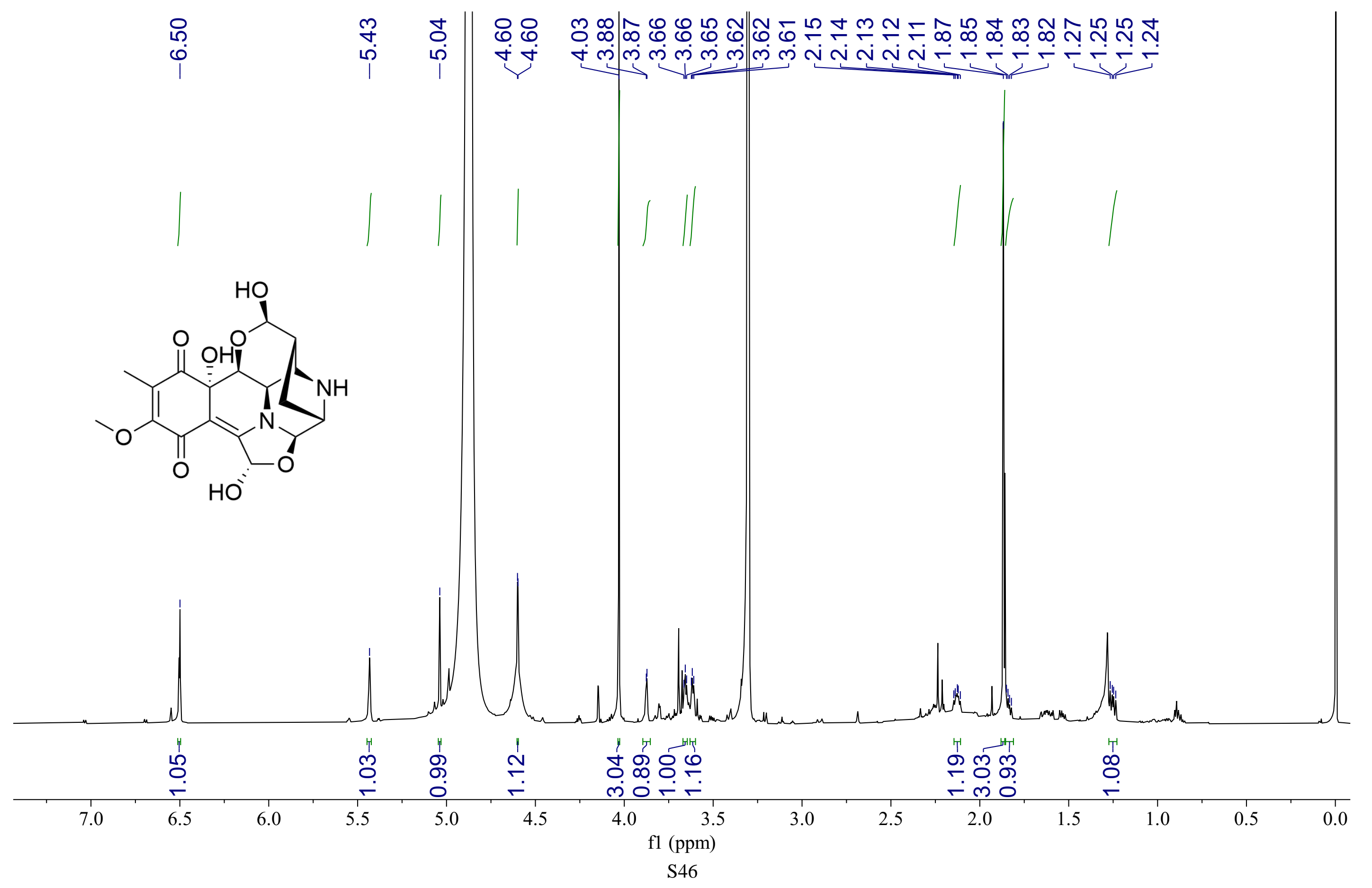


Figure S42. ${ }^{13} \mathrm{C}\left\{{ }^{1} \mathrm{H}\right\} \mathrm{NMR}(175 \mathrm{MHz})$ spectrum of 9 in $\mathrm{CD}_{3} \mathrm{OD}$

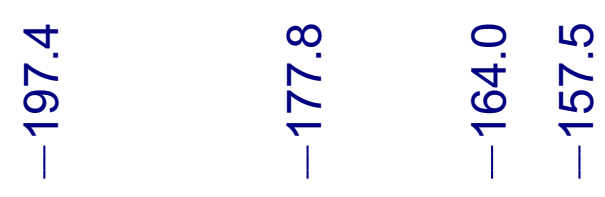

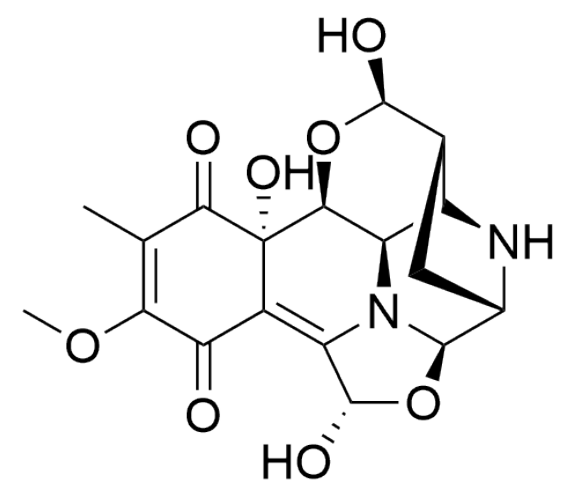

เ

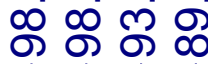

$\stackrel{\infty}{\stackrel{\infty}{*}}$

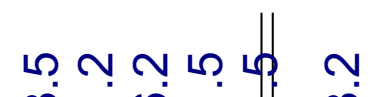

गु-

$1<1$

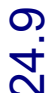

$\stackrel{\text { ก }}{2}$

ণ্

i

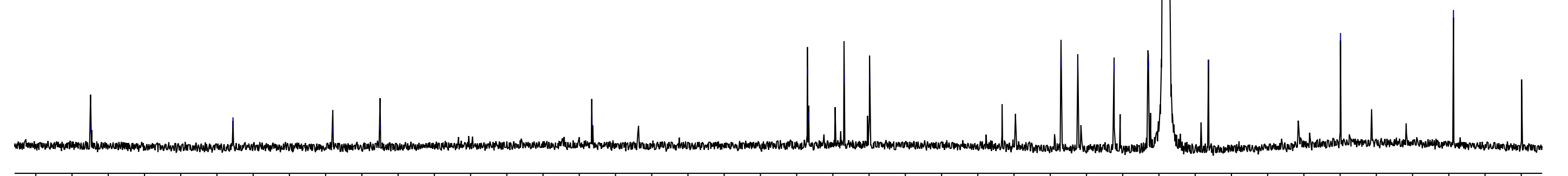




\section{Figure S43. HMQC spectrum of 9 in $\mathrm{CD}_{3} \mathrm{OD}$}

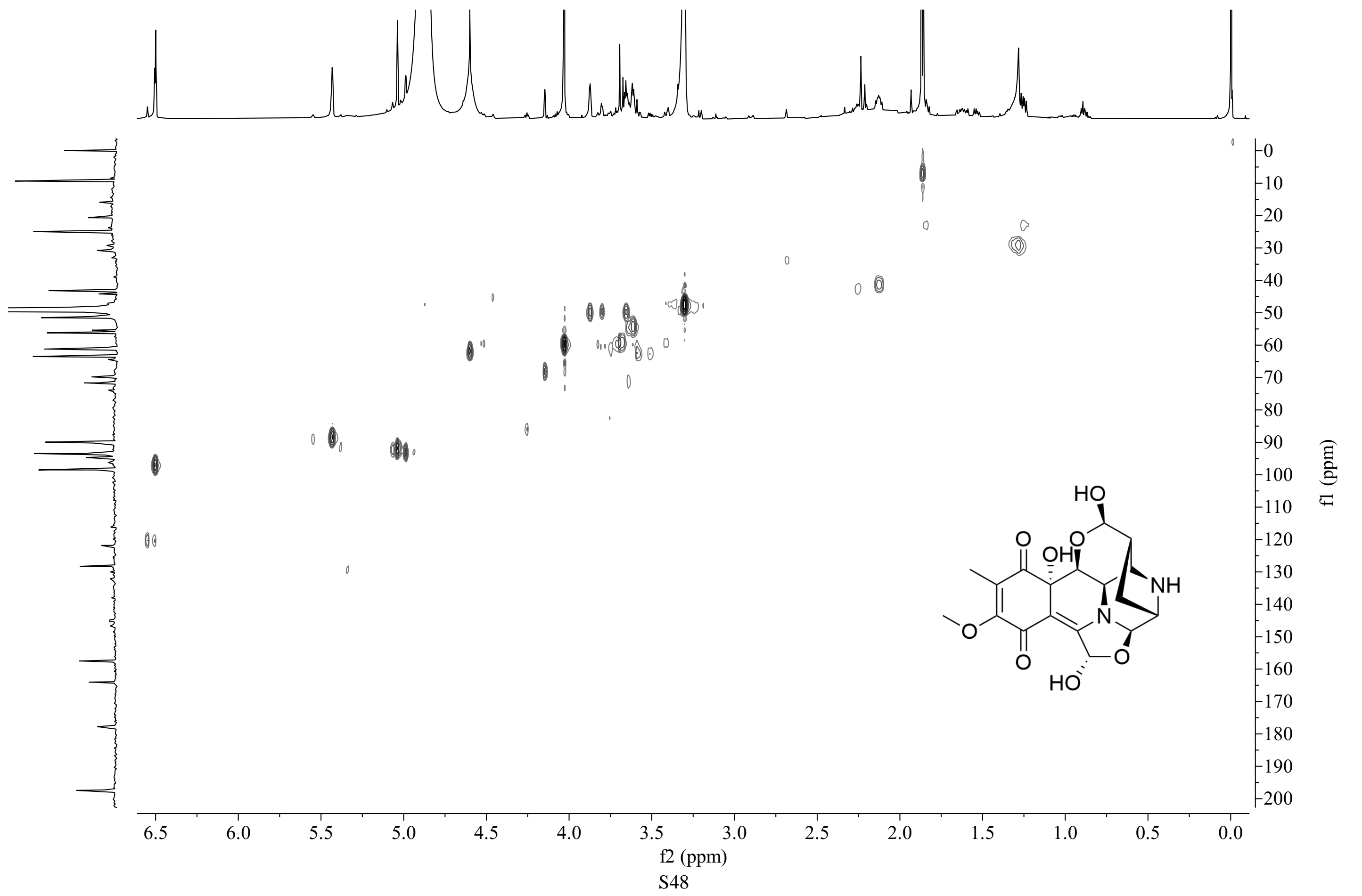


Figure S44. ${ }^{1} \mathrm{H}-{ }^{1} \mathrm{H}$ COSY spectrum of 9 in $\mathrm{CD}_{3} \mathrm{OD}$

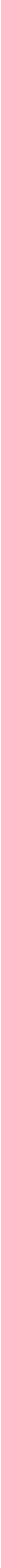


Figure S45. $\mathrm{HMBC}$ spectrum of 9 in $\mathrm{CD}_{3} \mathrm{OD}$

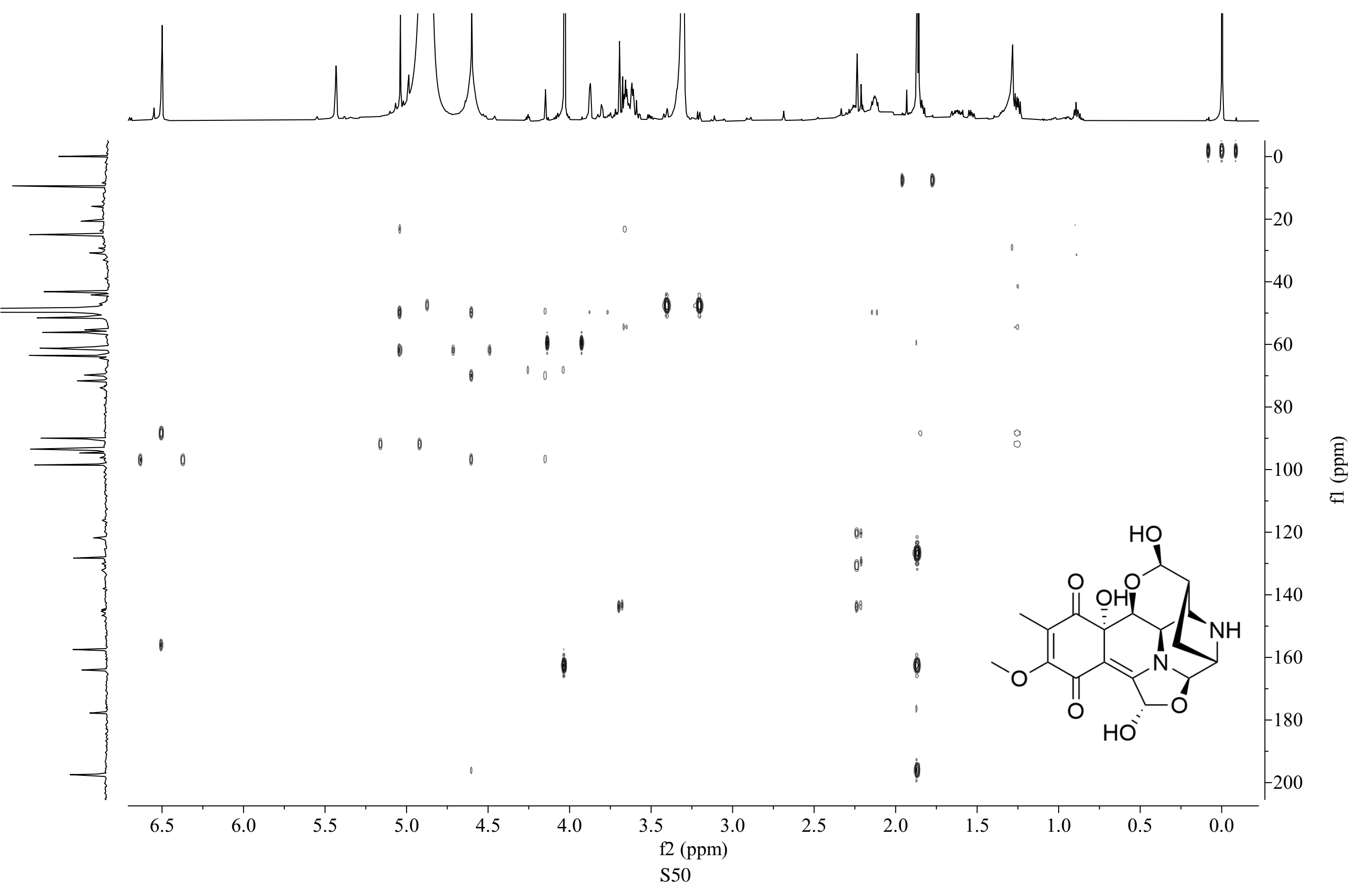


Figure S46. NOESY spectrum of 9 in $\mathrm{CD}_{3} \mathrm{OD}$

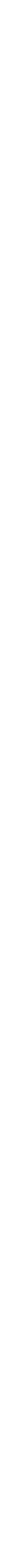


Figure S47. ${ }^{1} \mathrm{H}$ NMR $(700 \mathrm{MHz})$ spectrum of 10 in $\mathrm{CD}_{3} \mathrm{OD}$

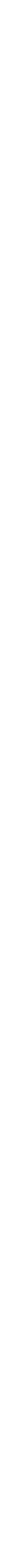


Figure S48. ${ }^{13} \mathrm{C}\left\{{ }^{1} \mathrm{H}\right\}$ NMR (175 MHz) spectrum of 10 in $\mathrm{CD}_{3} \mathrm{OD}$

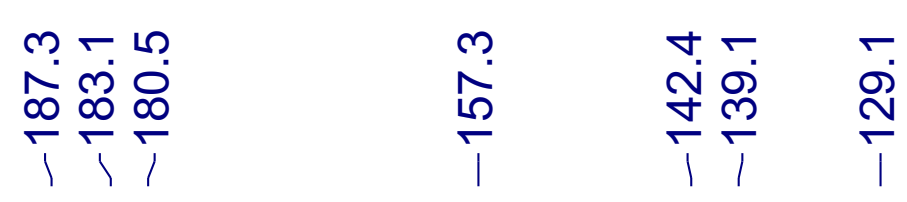<smiles>COC1=C(C)C(=O)C2=C(C1=O)[C@@H](CO)N1C[C@H]3C[C@H](N2)[C@H](CC3=O)C1</smiles>

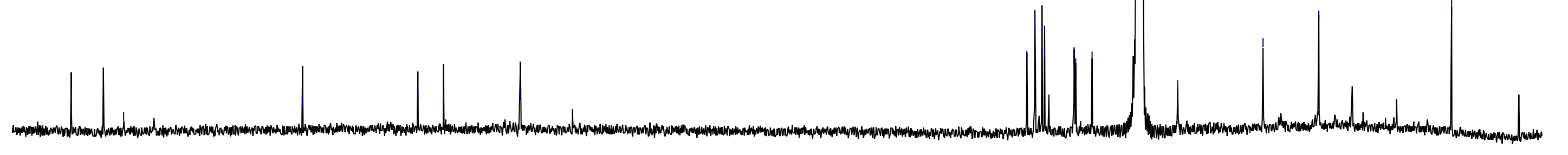


Figure S49. $\mathrm{HMQC}$ spectrum of 10 in $\mathrm{CD}_{3} \mathrm{OD}$

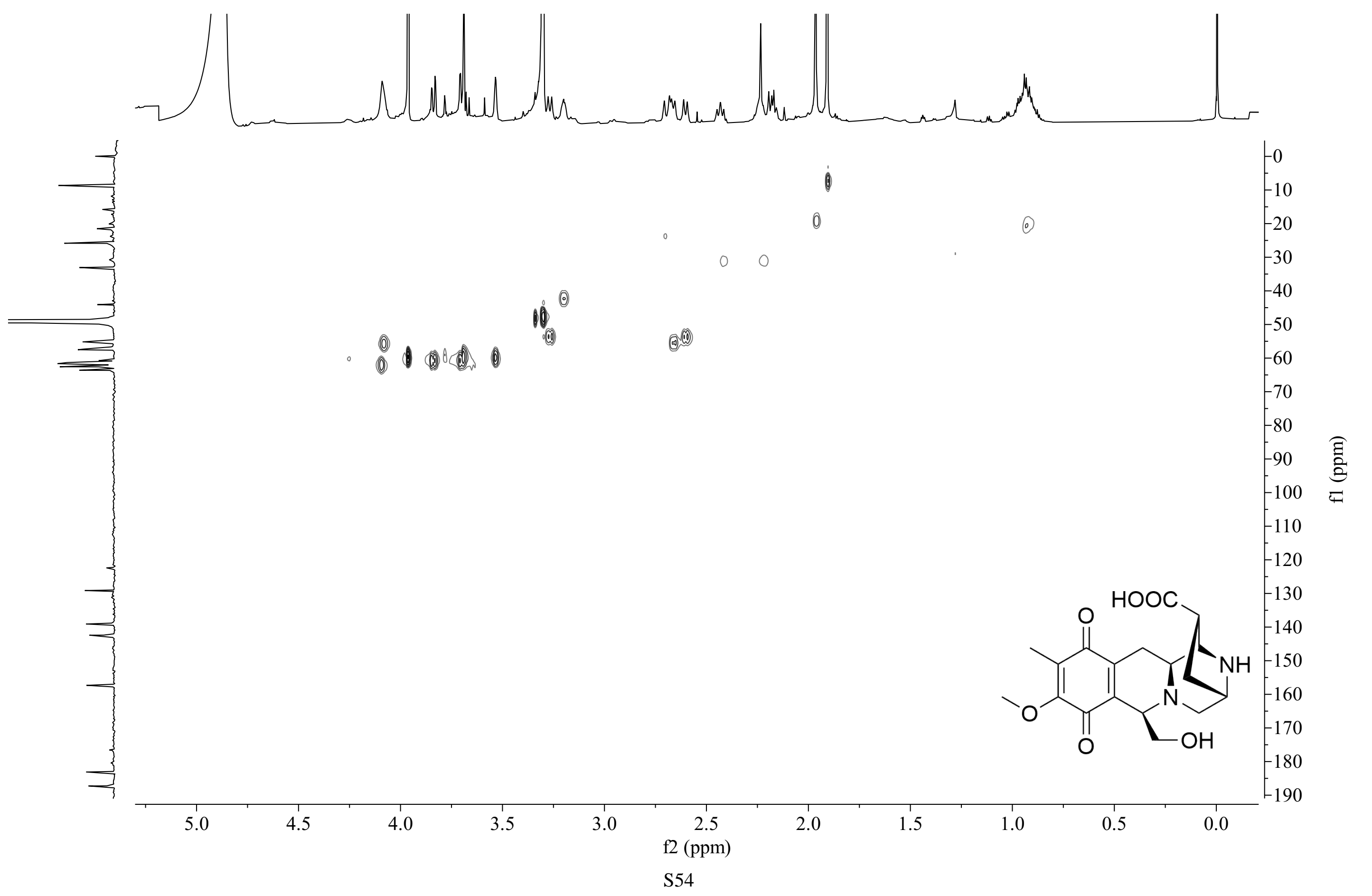


Figure S50. ${ }^{1} \mathrm{H}-{ }^{-1} \mathrm{H}$ COSY spectrum of 10 in $\mathrm{CD}_{3} \mathrm{OD}$

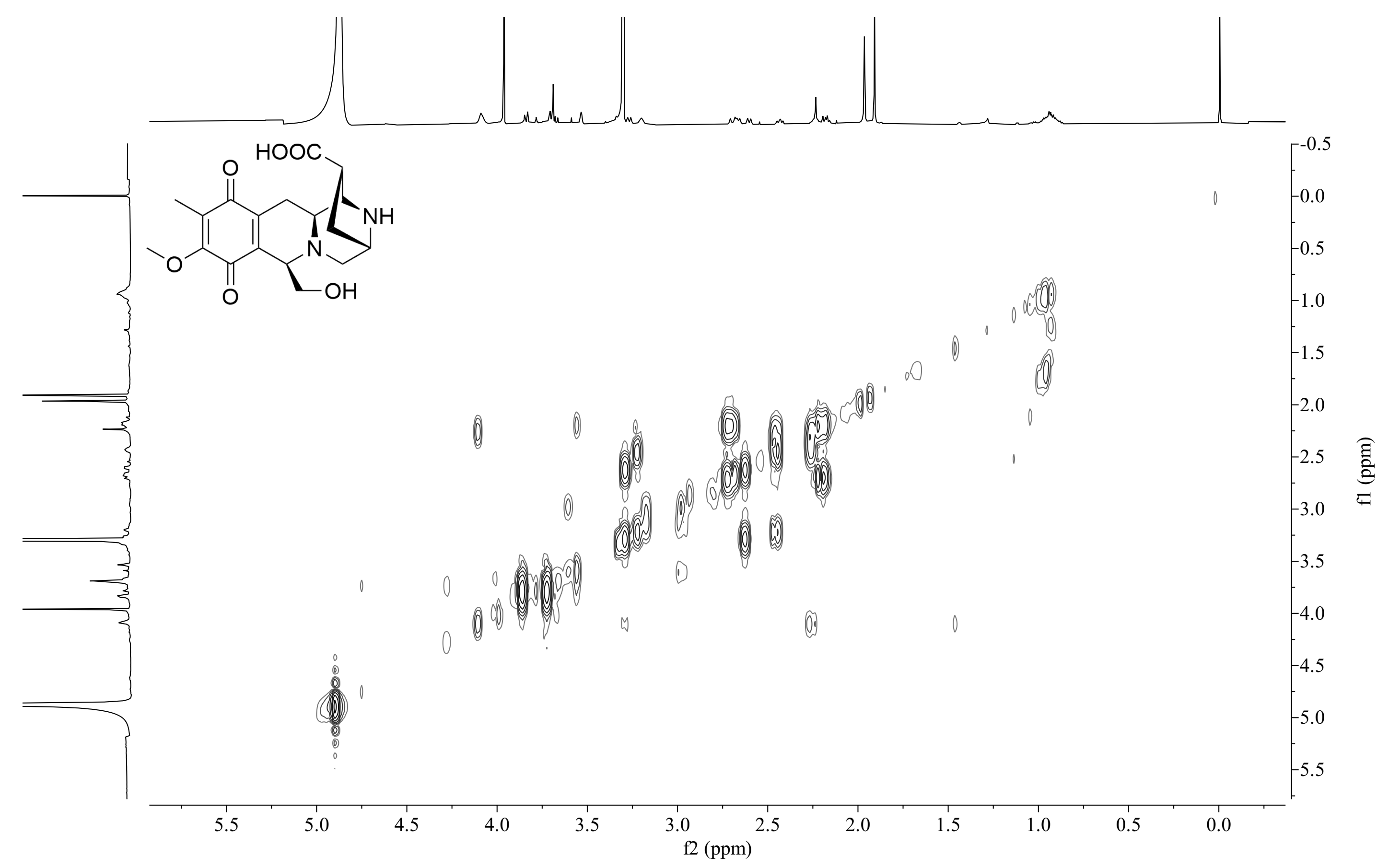


Figure S52. NOESY spectrum of $\mathbf{1 0}$ in $\mathrm{CD}_{3} \mathrm{OD}$

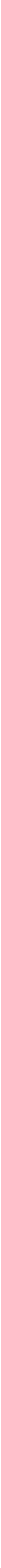


Figure S53. ${ }^{1} \mathrm{H}$ NMR $(700 \mathrm{MHz})$ spectrum of 11 in $\mathrm{CD}_{3} \mathrm{OD}$

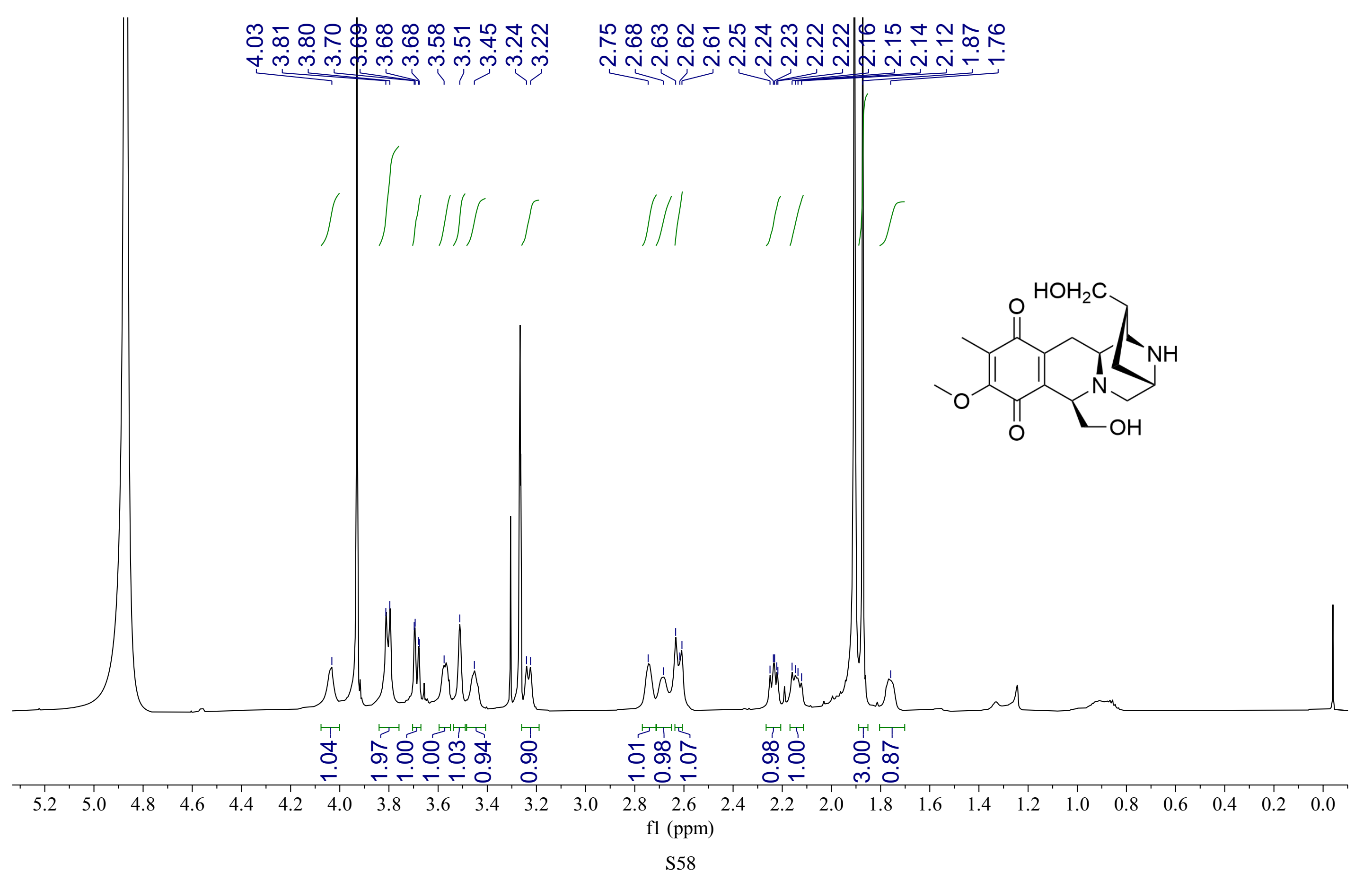


Figure S54. ${ }^{13} \mathrm{C}\left\{{ }^{1} \mathrm{H}\right\}$ NMR (175 MHz) spectrum of 11 in $\mathrm{CD}_{3} \mathrm{OD}$

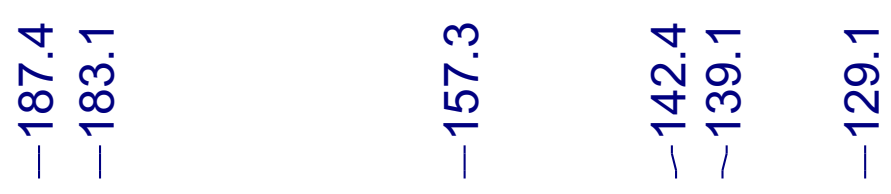

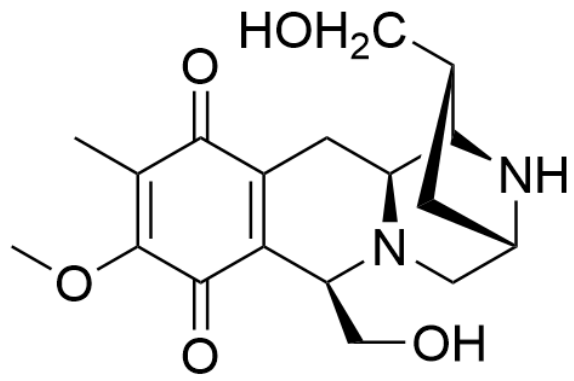

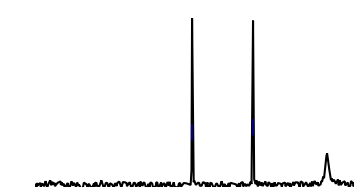

190

180

170

160

150

140

130

120

110 
Figure S55. HMQC spectrum of $\mathbf{1 1}$ in $\mathrm{CD}_{3} \mathrm{OD}$

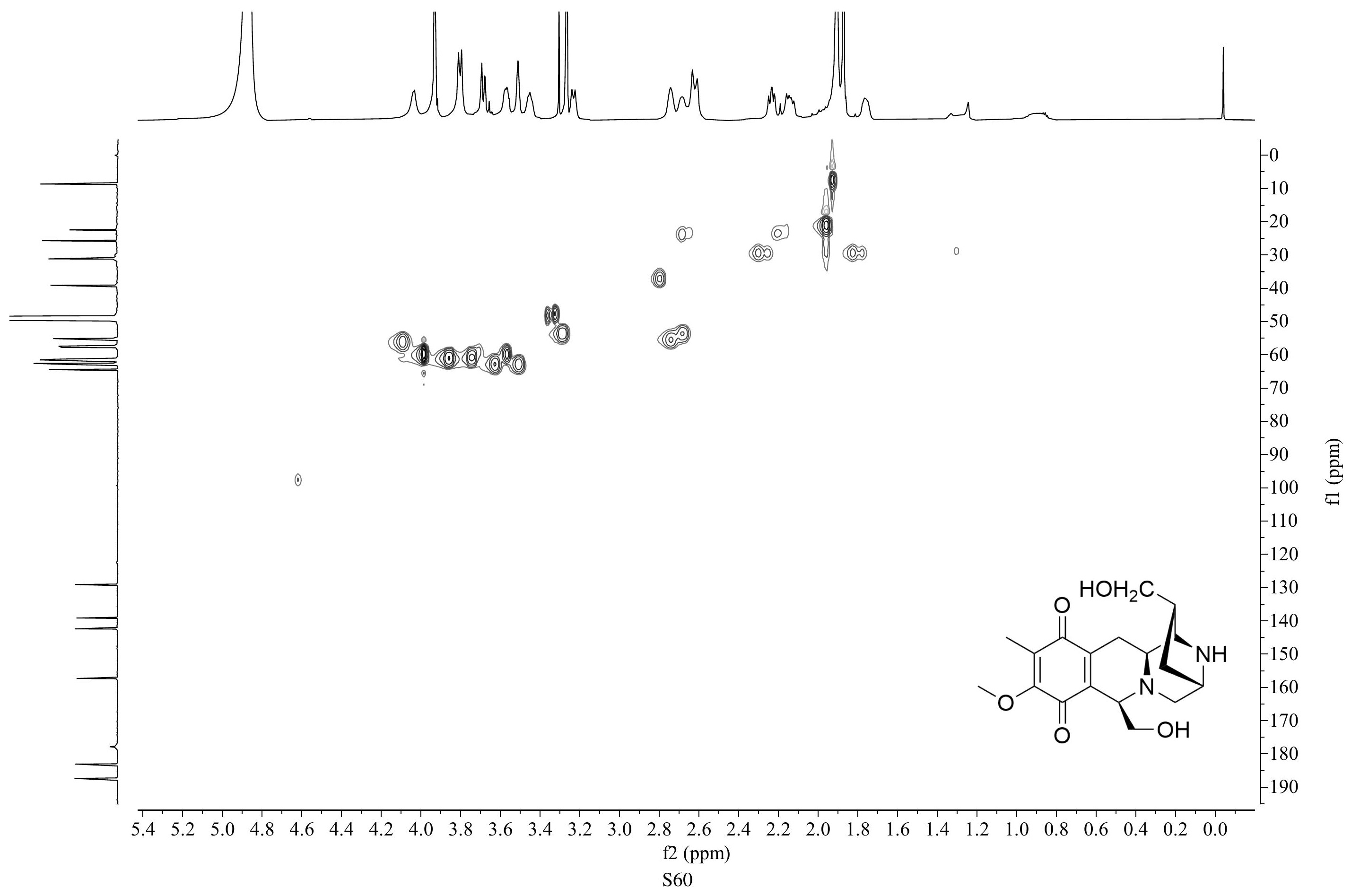


Figure 556. ${ }^{1} \mathrm{H}-{ }^{-1} \mathrm{H}$ COSY spectrum of $\mathbf{1 1}$ in $\mathrm{CD}_{3} \mathrm{OD}$

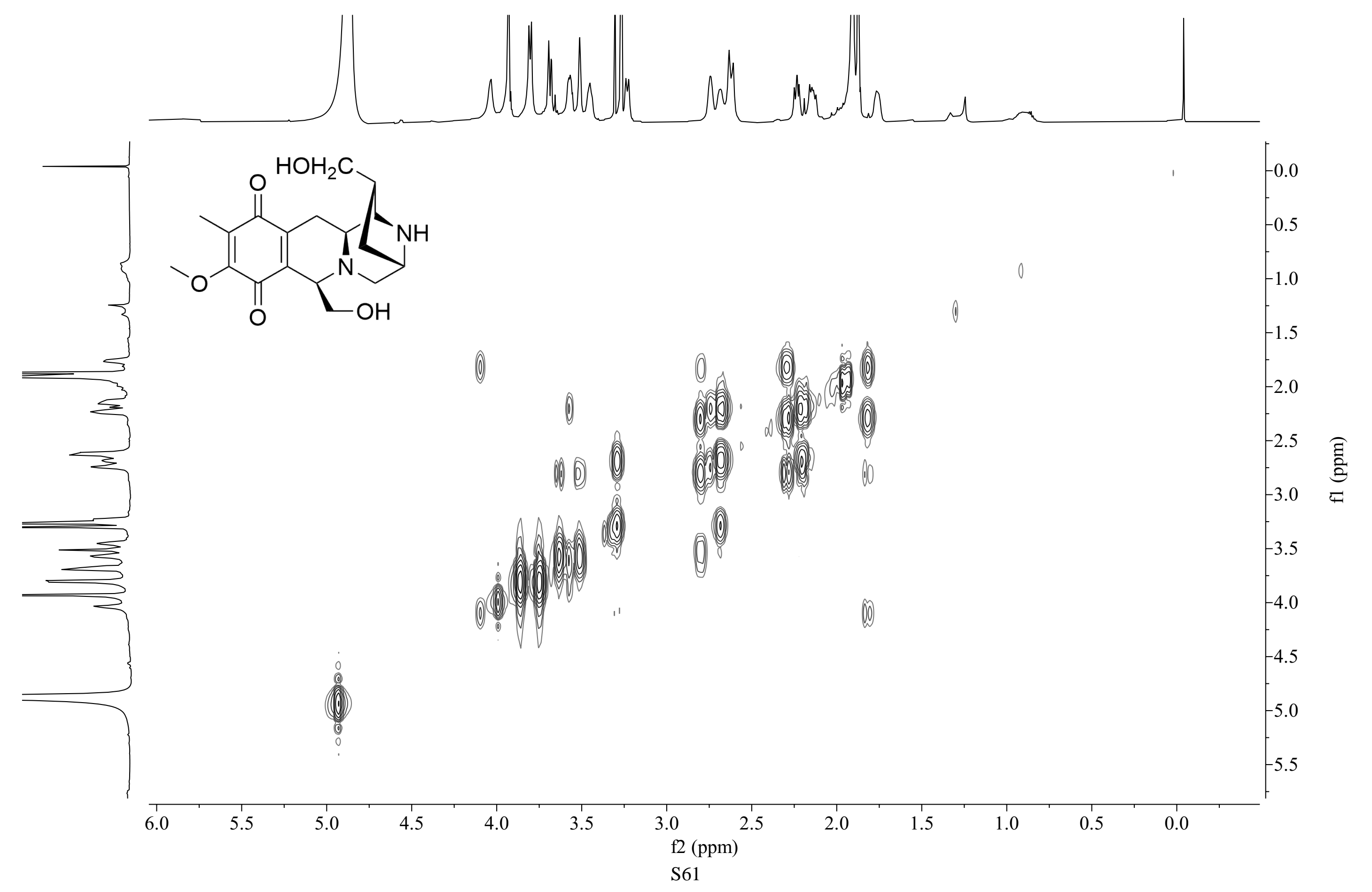


Figure S57. $\mathrm{HMBC}$ spectrum of $\mathbf{1 1}$ in $\mathrm{CD}_{3} \mathrm{OD}$

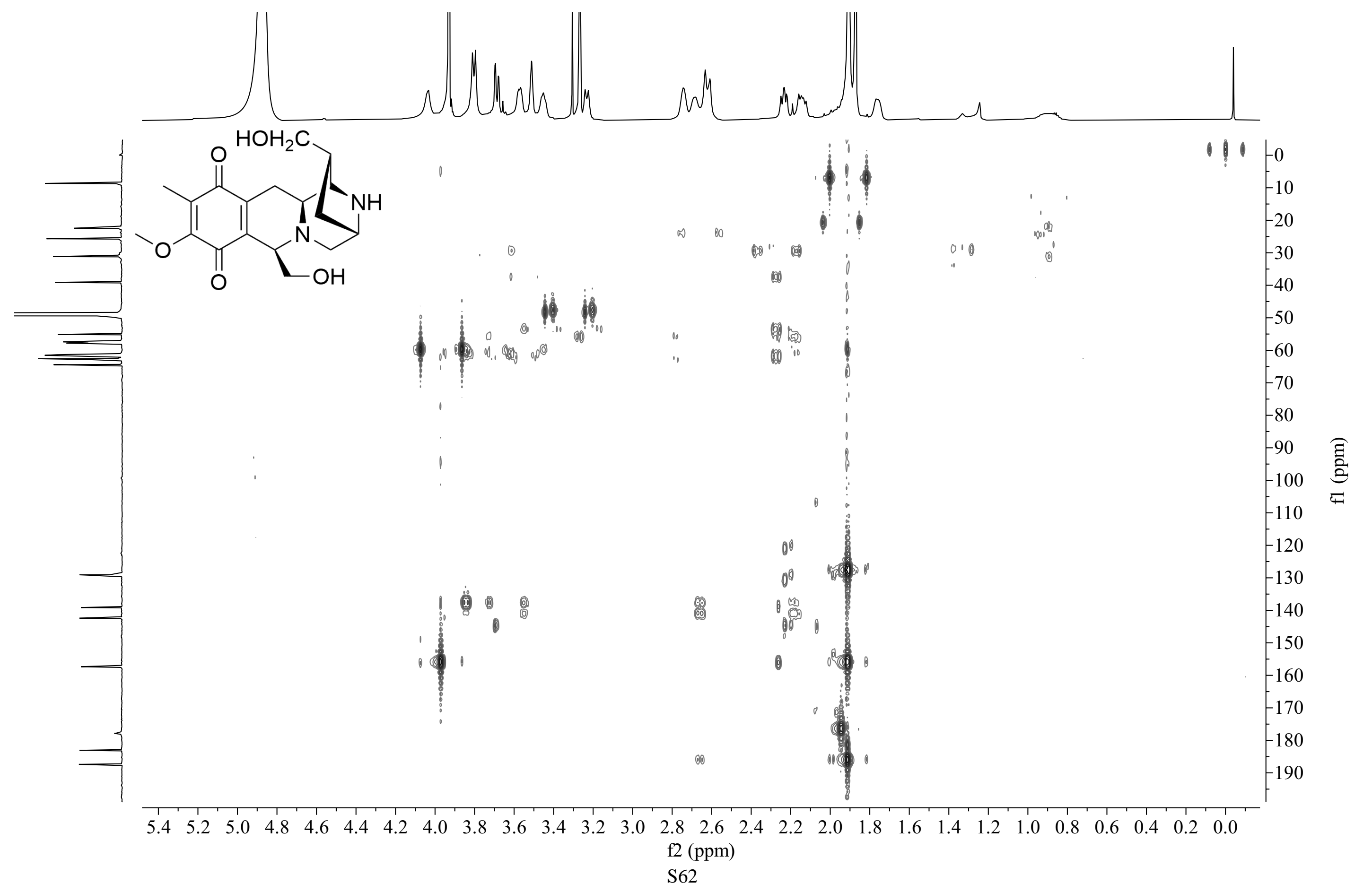


Figure S58. NOESY spectrum of $\mathbf{1 1}$ in $\mathrm{CD}_{3} \mathrm{OD}$

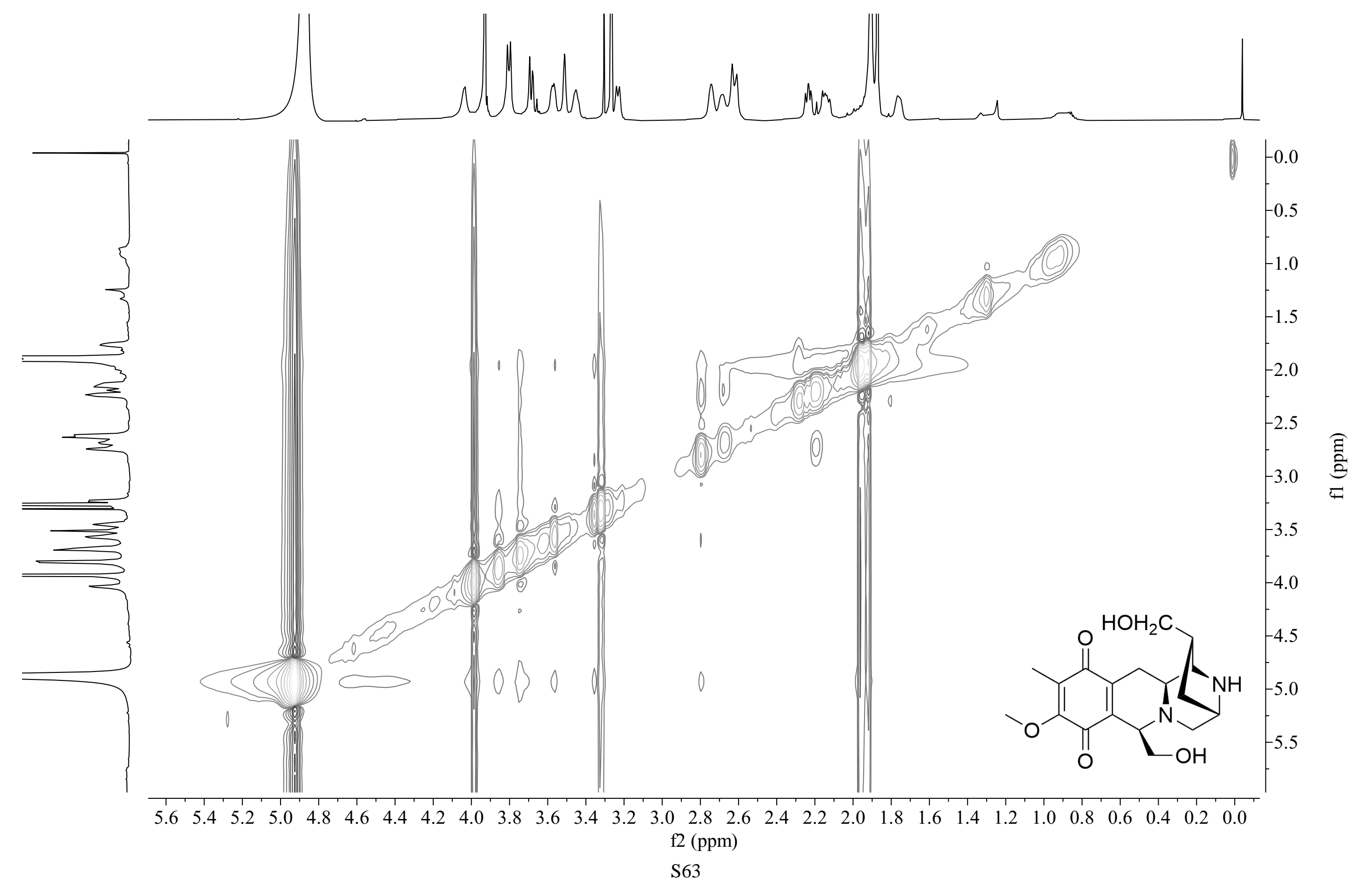

